\title{
Lattice Boltzmann modeling and simulation of compressible flows
}

\author{
Aiguo Xu ${ }^{1 *}$, Guangcai Zhang ${ }^{1}$, Yanbiao $\mathrm{Gan}^{2}$, Feng $\mathrm{Chen}^{3}$, Xijun $\mathrm{Yu}^{1}$ \\ 1 National Key Laboratory of Computational Physics, \\ Institute of Applied Physics and Computational Mathematics, \\ P. O. Box 8009-26, Beijing 100088, P.R.China \\ ${ }^{2}$ North China Institute of Aerospace Engineering, Langfang 065000, P.R.China \\ 3 School of science, Linyi University, Linyi 276005, P. R. China
}

(Dated: September 8, 2018)

\begin{abstract}
In this mini-review we summarize the progress of Lattice Boltzmann(LB) modeling and simulating compressible flows in our group in recent years. Main contents include (i) Single-RelaxationTime(SRT) LB model supplemented by additional viscosity, (ii) Multiple-Relaxation-Time(MRT) LB model, and (iii) LB study on hydrodynamic instabilities. The former two belong to improvements of physical modeling and the third belongs to simulation or application. The SRT-LB model supplemented by additional viscosity keeps the original framework of Lattice Bhatnagar-GrossKrook (LBGK). So, it is easier and more convenient for previous SRT-LB users. The MRT-LB is a completely new framework for physical modeling. It significantly extends the range of LB applications. The cost is longer computational time. The developed SRT-LB and MRT-LB are complementary from the sides of convenience and applicability.
\end{abstract}

PACS numbers: 47.11.-j, 47.55.-t, 05.20.Dd

\footnotetext{
* Corresponding author. Email address: Xu_Aiguo@iapcm.ac.cn
} 


\section{INTRODUCTION}

During the past two decades the lattice Boltzmann (LB) method has emerged as a competitive scheme for simulating various nearly incompressible complex flows [1], ranging from magnetohydrodynamics [2, 3]], to flows of suspensions [4], flows with phase separation [5-14], flows through porous media [15, 16], etc. With increasing the Mach number, the compressibility of flow becomes more pronounced. Such high speed compressible flows are ubiquitous in explosion physics, aerophysics and astrophysics, etc. Up to now, the LB modeling and simulating of compressible flows, especially those with shocks and/or discontinuities, is still a challenging issue.

Given the great importance of shocking and detonation in many fields of physics and engineering [17 19], constructing LB models for high speed compressible flows has been attempted since the early days of LB research [1]. To proceed, we first discuss the most fundamental problem "what is LB ?". The views are not exactly the same in papers by different authors. Since having different knowledge backgrounds and working in different fields, different authors may use LB to solve different problems and focus on different sides of LB. Understandably, even for the same author, the views will be updated with extending research experience. Globally speaking, the views on LB can be classified into two categories. The first category regards LB as a new scheme for simulating hydrodynamic equations such as the Euler equations and Navier-Stokes equations. The second category regards LB as a kind of new model of physical systems. Physical model construction and numerical method design are the first two steps for numerical study on any physical problems. Compared with numerical methods, the physical model construction is the first step and more fundamental. Only after the physical model is fixed can the corresponding numerical method be established. Clearly, the first kind of view starts LB research from the second step, numerical method design. It does not consider the improvements of the physical modeling. In other words, it assumes that the original hydrodynamic equations are sufficiently exact for modeling the problem under consideration. The second kind of view puts LB research on the more fundamental step, physical modeling. For this view the numerical method is the second important issue. It accepts any reasonable numerical methods no matter they are new or traditional. The second kind of view aims at physical problems. The point of the second view is that, compared with the traditional hydrodynamic equations, the LB framework contains 
more physical components. The theoretical reasons are as below. The LB model is based on the Boltzmann equation which is one of the most fundamental equations in non-equilibrium statistical physics. It naturally inherits some intrinsic characteristics of the latter. According to the Chapman-Enskog analysis, one can expand the distribution function around its equilibrium as Taylor series in the Knudsen number. When the Knudsen number approaches zero, the system is nearly in equilibrium state, the deviation from equilibrium is negligible, the LB model corresponds to or recovers the Euler equations. When the first order terms in Knudsen number have to be accounted and the second order terms are negligible, in other words, when the system slightly deviates from the equilibrium, the LB model corresponds to or recovers the Navier-Stokes equations. When the system deviates more from equilibrium and the second order terms in Knudsen number have to be taken into account, the LB model is beyond the Navier-Stokes description. The theoretical framework of LB is self-adaptive for describing complex systems where the deviations from equilibrium are spatially and temporally varying. From the view of modeling precision on detailed dynamics, it is less than Molecular Dynamics(MD). It adopts the concept of distribution function. It is generally considered as a kind of mesoscopic modeling. For continuum system, the LB should give the same results as those of hydrodynamics equations. For non-continuum systems such as the boundary layers where the Knudsen number is high, the LB should give the same results as those of other mature methods such as MD or Monte Carlo(MC). In between the two kinds of limiting cases, the hydrodynamic equations are not valid, the MD and MC are reasonable but not practical due to the huge quantity of computations. For such cases, the LB modeling and simulation still work. Its results should be checked by physical principles and analyses. Just as in traditional Computational Fluid Dynamics(CFD) where different discretization schemes work for different problems, for different systems one should compose or choose different LB models.

In 1992 Alexander et al [20] proposed a compressible LB model where the main skill is to introduce a flexible sound speed so that the Mach number may become higher. This model works only for nearly isothermal compressible systems. In 1999 Yan et al [21] proposed a LB scheme for compressible Euler equations. In this model a Discrete Velocity Model(DVM) with three energy levels is used. Sun et al [22, 23] proposed an adaptive LB model where the particle velocities vary with the Mach number and internal energy. The model partly frees the particle velocity from fixed values. It works for more extensive systems compared with 
previous LB versions. Its two-dimensional and three-dimensional versions were published in 1998 and 2003, respectively. The evolutions of all those models follow the traditional "propagation + collision" mode. All of them belong to the standard LB models. Due to the inconvenience of application and/or numerical instability problems, few physical results based on those models can be found.

For modeling and simulating compressible flows, an alternative way is to use the FiniteDifference(FD)-LB method. Tsutahara group [24 28] in Kobe university proposed several FD-LB models in recent years. The FD-LB model frees the combination of spatial and temporal discretizations. The sizes of particle velocities are flexible. So it is much more convenient to meet the requirements for simulating compressible fluids. The FD-LB scheme was then extended to the case of binary fluids [29, 30]. But numerical instability problem blocks its practical applications to systems with a Mach number being larger than 1. In fact, as for the numerical instability problem, many attempts have been made. Typical examples are referred to the entropy LB model [31, 32], FIX-UP scheme[33], flux-limters approach[34], etc. But most of the discussions were still focused on systems with small Mach numbers.

To model and simulate high speed compressible flows, especially those with shocks, our group developed two schemes in recent years. The first is to introduce additional viscosity and improve the discretization of spatial and temporal derivatives [35 40]. This scheme does not change the framework of the original LB model. The second is to develop Multiple Relaxation Time(MRT) LB models [41-45]. The framework is changed in the second scheme. The first scheme is based on the following facts. (i) The numerical fluid particles do not distinguish the original viscosity and additional viscosity. (ii) Introducing additional viscosity is equivalent to modifying the relaxation time from some sense. (iii) Better template of discretization may damp the numerical anisotropy. Our improved models work for both high speed and low speed flows. So, they make it possible to simulate stable shocks in compressible fluids. The first scheme is based on the original Bhatnagar-Gross-Krook(BGK) model. It is a remedy under the original framework.

The rest of the paper is structured as follows. We first introduce a few improved LB models based on the first scheme in section II. The MRT scheme is reviewed in section III. Section IV shows two typical applications, LB studies on Richtmyer-Meshkov(RM) and Kelvin-Helmhotz(KH) instabilities. Section V summarizes the present paper. 


\section{SRT MODEL SUPPLEMENTED BY ADDITIONAL VISCOSITY}

Among the two-dimensional FD-LB models for compressible flows, the one by Kataoka and Tsutahara [24] is typical. It has very simple and strict theoretical background, uses a DVM with only 9 components. The specific heat ratio is flexible. But the numerical instability blocks its application in supersonic flows. Therefore, our first LB model for high speed compressible flows is created by improving the Kataoka-Tsutahara(KT) model.

The LB kinetic equation with BGK approximation reads,

$$
\frac{\partial f_{i}}{\partial t}+v_{i \alpha} \frac{\partial f_{i}}{\partial x_{\alpha}}=\frac{1}{\tau}\left[f_{i}^{e q}-f_{i}\right]
$$

where $f_{i}\left(f_{i}^{e q}\right)$ is the discrete (equilibrium) distribution function; $\mathbf{v}_{i}$ is the $i$-th discrete velocity, $i=0, \cdots, N-1 ; N$ is the total number of the discrete velocity; index $\alpha=1,2$, 3 corresponding to $x, y$, and $z$, respectively; $\tau$ is the relaxation time determining the speed of approaching equilibrium. Sometimes, $\tau$ is rewritten as $\epsilon \tau^{\prime}$, where $\epsilon$ is a dimensionless number, the Knudsen number. The original KT model corresponds to the complete Euler equations

$$
\begin{aligned}
\frac{\partial \rho}{\partial t}+\frac{\partial\left(\rho u_{\alpha}\right)}{\partial x_{\alpha}} & =0, \\
\frac{\partial\left(\rho u_{\alpha}\right)}{\partial t}+\frac{\partial\left(\rho u_{\alpha} u_{\beta}\right)}{\partial x_{\beta}}+\frac{\partial P}{\partial x_{\alpha}} & =0, \\
\frac{\partial}{\partial t}\left(E+\frac{1}{2} \rho u_{\alpha}^{2}\right)+\frac{\partial}{\partial x_{\alpha}}\left[u_{\alpha}\left(E+\frac{1}{2} \rho u_{\beta}^{2}+P\right)\right] & =0,
\end{aligned}
$$

when the knudsen number $\epsilon$ approaching zero. Here $\rho, u, P(=\rho T), E(=\rho T /(\gamma-1))$ are the hydrodynamic density, flow velocity, pressure and internal energy, respectively; $T$ is the temperature and $\gamma$ is the specific-heat ratio. To make $\gamma$ flexible, a constant, $b=2 /(\gamma-1)$, is introduced. The following constraints are needed for this model,

$$
\begin{gathered}
\rho=\sum_{i=0}^{N-1} f_{i}^{e q}=\sum_{i=0}^{N-1} f_{i}, \\
\rho u_{\alpha}=\sum_{i=0}^{N-1} f_{i}^{e q} v_{i \alpha}=\sum_{i=0}^{N-1} f_{i} v_{i \alpha} \\
\rho\left(b R T+u_{\alpha}^{2}\right)=\sum_{i=0}^{N-1} f_{i}^{e q}\left(v_{i \alpha}^{2}+\eta_{i}^{2}\right)=\sum_{i=0}^{N-1} f_{i}\left(v_{i \alpha}^{2}+\eta_{i}^{2}\right),
\end{gathered}
$$




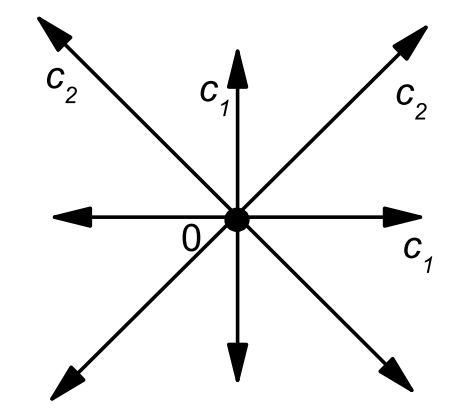

FIG. 1: Schematic figure of the discrete velocity model.

$$
\begin{gathered}
P \delta_{\alpha \beta}+\rho u_{\alpha} u_{\beta}=\sum_{i=0}^{N-1} f_{i}^{e q} v_{i \alpha} v_{i \beta}, \\
\rho\left[(b+2) R T+u_{\beta}^{2}\right] u_{\alpha}=\sum_{i=0}^{N-1} f_{i}^{e q}\left(v_{i \beta}^{2}+\eta_{i}^{2}\right) v_{i \alpha},
\end{gathered}
$$

where $\eta_{i}$ is another variable introduced to make specific-heat ratio flexible.

In the two-dimensional case, the KT DVM has nine components. It reads

$$
\begin{gathered}
\left(v_{i 1}, v_{i 2}\right)= \\
\begin{cases}(0,0), & i=0 \\
c_{1}\left[\cos \left(\frac{\pi(i+1)}{2}\right), \sin \left(\frac{\pi(i+1)}{2}\right)\right], & i=1,2,3,4 \\
c_{2}\left[\cos \pi\left(\frac{i+1}{2}+\frac{1}{4}\right), \sin \pi\left(\frac{i+1}{2}+\frac{1}{4}\right)\right], & i=5,6,7,8\end{cases} \\
\eta_{i}=\left\{\begin{array}{ll}
\eta_{0}, & i=0 \\
0, & i=1,2, \ldots, 8
\end{array} .\right.
\end{gathered}
$$

A schematic figure of the distribution of the discrete velocities is shown in Fig.1, where $c_{1}$ and $c_{2}$ are constants which should not depart faraway from the flow velocity $u . c_{2}$ is generally chosen $1.0 \sim 3.0$ times of $c_{1}$.

The local equilibrium distribution function is computed by

$$
f_{i}^{e q}=\rho\left(A_{i}+B_{i} v_{i \alpha} u_{\alpha}+D_{i} u_{\alpha} v_{i \alpha} u_{\beta} v_{i \beta}\right), i=0,1, \cdots, 8,
$$

where

$$
A_{i}= \begin{cases}\frac{b-2}{\eta_{0}^{2}} T, & i=0 \\ \frac{1}{4\left(c_{1}^{2}-c_{2}^{2}\right)}\left[-c_{2}^{2}+\left((b-2) \frac{c_{2}^{2}}{\eta_{0}^{2}}+2\right) T+\frac{c_{2}^{2}}{c_{1}^{2}} u_{\alpha}^{2}\right], & i=1,2,3,4 \\ \frac{1}{4\left(c_{2}^{2}-c_{1}^{2}\right)}\left[-c_{1}^{2}+\left((b-2) \frac{c_{1}^{2}}{\eta_{0}^{2}}+2\right) T+\frac{c_{1}^{2}}{c_{2}^{2}} u_{\alpha}^{2}\right], & i=5,6,7,8\end{cases}
$$




$$
B_{i}= \begin{cases}0, & i=0 \\
\frac{-c_{2}^{2}+(b+2) T+u_{\beta}^{2}}{2 c_{1}^{2}\left(c_{1}^{2}-c_{2}^{2}\right)}, & i=1,2,3,4, D_{i}=\left\{\begin{array}{ll}
0, & i=0 \\
\frac{-c_{1}^{2}+(b+2) T+u_{\beta}^{2}}{2 c_{2}^{2}\left(c_{2}^{2}-c_{1}^{2}\right)}, & i=5,6,7,8
\end{array} \frac{1}{2 c_{1}^{4},} \quad i=1,2,3,4\right. \\
\frac{1}{2 c_{2}^{4}}, & i=5,6,7,8\end{cases}
$$

Parameters $\eta_{0}, c_{1}$ and $c_{2}$ are independent in this DVM. $\eta_{0}$ influences $f_{i}^{e q}$ via the expansion coefficient $A_{i}$. In the original KT model, the usual FD scheme with first-order forward in time and second-order upwinding in space is used.

To make practical the LB simulation to the supersonic flows, we propose an alternative FD scheme combined with an additional dissipation term to overcome the numerical instability problem. The LB equation (1) can be regarded as non-dimensional. In this work, we consider $\tau=\epsilon \tau^{\prime}$ and set the time step $\Delta t$ to be numerically equal to the Knudsen number $\varepsilon$. Thus, from Eq.(11) we have

$$
f_{i}(\mathbf{x}, t+\Delta t)-f_{i}(\mathbf{x}, t)+v_{i \alpha} \frac{\partial f_{i}(\mathbf{x}, t)}{\partial x_{\alpha}} \Delta t=\frac{1}{\tau}\left[f_{i}^{e q}(\mathbf{x}, t)-f_{i}(\mathbf{x}, t)\right]
$$

In Eq.(13) $\tau^{\prime}$ has been written as $\tau$ for simplicity. The spatial derivative $\partial f_{i} / \partial x$ can be calculated by

$$
\begin{aligned}
& \text { If } v_{i x} \geq 0, \quad \frac{\partial f_{i}}{\partial x}=\frac{\beta f_{i}(x+\Delta x, t)+(1-2 \beta) f_{i}(x, t)-(1-\beta) f_{i}(x-\Delta x, t)}{\Delta x} \\
& \text { If } v_{i x}<0, \quad \frac{\partial f_{i}}{\partial x}=\frac{(1-\beta) f_{i}(x+\Delta x, t)-(1-2 \beta) f_{i}(x, t)-\beta f_{i}(x-\Delta x, t)}{\Delta x}
\end{aligned}
$$

In Eqs.(14) and (15), $0 \leq \beta \leq 0.5$. If $\beta$ takes zero, then they are no other than the first-order upwind scheme in space; if $\beta$ takes 0.5 , they recover to the general central difference scheme. $\partial f_{i} / \partial y$ can be calculated in a similar way. Actually, Eqs.(14) and (15) can be rewritten as

$$
\begin{aligned}
\text { If } v_{i x} \geq & 0, \quad \frac{\partial f_{i}}{\partial x}=\frac{f_{i}(x, t)-f_{i}(x-\Delta x, t)}{\Delta x} \\
& +\frac{\beta \Delta x\left[f_{i}(x+\Delta x, t)+f_{i}(x-\Delta x, t)-2 f_{i}(x, t)\right]}{\Delta x^{2}} ; \\
\text { If } v_{i x}< & 0, \quad \frac{\partial f_{i}}{\partial x}=\frac{f_{i}(x+\Delta x, t)-f_{i}(x, t)}{\Delta x} \\
& -\frac{\beta \Delta x\left[f_{i}(x+\Delta x, t)+f_{i}(x-\Delta x, t)-2 f_{i}(x, t)\right]}{\Delta x^{2}} .
\end{aligned}
$$

The second terms in the Right-Hand-Side(RHS) of Eqs.(16) and (17) can be regarded as some kind of additional viscosities which can reduce some unphysical phenomena such as 
wall-heating, but they are not enough. Additional dissipation term is needed. The final LB equation reads

$$
f_{i}(\mathbf{x}, t+\Delta t)-f_{i}(\mathbf{x}, t)+v_{i \alpha} \frac{\partial f_{i}(\mathbf{x}, t)}{\partial x_{\alpha}} \Delta t-\lambda_{i} \sum_{\alpha=1}^{2} \frac{\partial^{2} f_{i}(\mathbf{x}, t)}{\partial x_{\alpha}^{2}} \Delta t=\frac{1}{\tau}\left[f_{i}^{e q}(\mathbf{x}, t)-f_{i}(\mathbf{x}, t)\right]
$$

where $\lambda_{i}$ is a small number not varying in space or time. The second-order derivative $\frac{\partial^{2} f_{i}(\mathbf{x}, t)}{\partial x_{\alpha}^{2}}$ can be calculated by the central difference scheme. In our simulations $\Delta x=\Delta y$ and the parameter $\beta$ is generally chosen to be 0.25 if not particularly claimed. How to choose the $\lambda_{i}$ is the key problem. Analysis by the software, Mathematica, and numerical tests show that we can choose $\lambda_{i}$ around the following way,

$$
\lambda_{i}= \begin{cases}c_{1} \Delta x, & i=0 \\ c_{1} \Delta x / 10, & i=1,2,3,4 . \\ 0, & i=5,6,7,8\end{cases}
$$

The improved model is validated by well-known benchmark tests. Simulations on Riemann problems with very high ratios (1000:1) of pressure and density also show good accuracy and stability. Regular and double Mach shock reflections are successfully simulated. It should be commented that, since using constraint, $\Delta t=\epsilon$, such a model can only be regarded as a new scheme to simulate the Euler equations. The added viscosity terms can be regarded as a kind of slight remedy to the traditional hydrodynamic model.

In 2008 Gan, Xu, Zhang, et al [36] developed a LB model for high speed compressible flows. In this model, the constraint, $\Delta t=\epsilon$, is eliminated. Therefore, it can be regarded as a mesoscopic new model. In the continuum limit it corresponds to the Navier-Stokes equations. The model is composed of three components: (i) the DVM by Watari and Tsutahara [26], (ii) a modified Lax-Wendroff FD scheme where reasonable dissipation and dispersion are naturally included, (iii) additional viscosity. The improved model is convenient to compromise the high accuracy and stability. The included dispersion term can effectively reduce the numerical oscillation at discontinuity. Shock tubes and shock reflections are used to validate the new scheme. In our numerical tests the Mach numbers are successfully increased up to 20 or higher. In Fig. 2 we show a simulation result on double Mach reflection by the improved model. The initial pressure ratio here is high. A planar shock is incident towards an oblique surface with a $30^{\circ}$ angle to the direction of propagation of the shock. A uniform mesh size of $500 \times 200$ is used for the numerical simulation. The conditions for both 


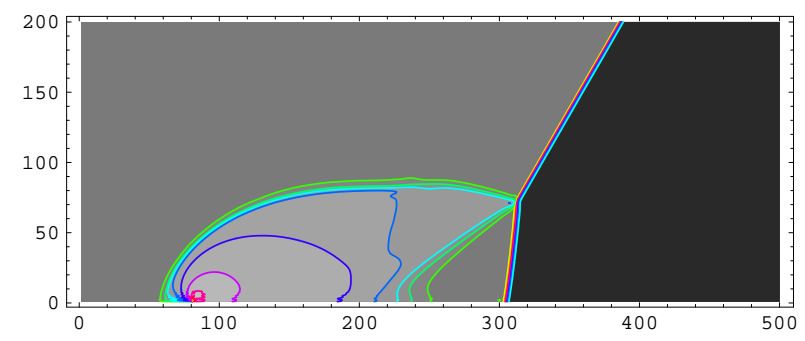

(a)

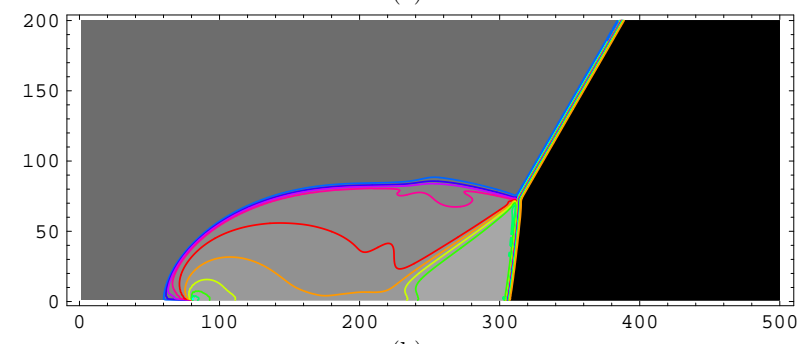

(b)

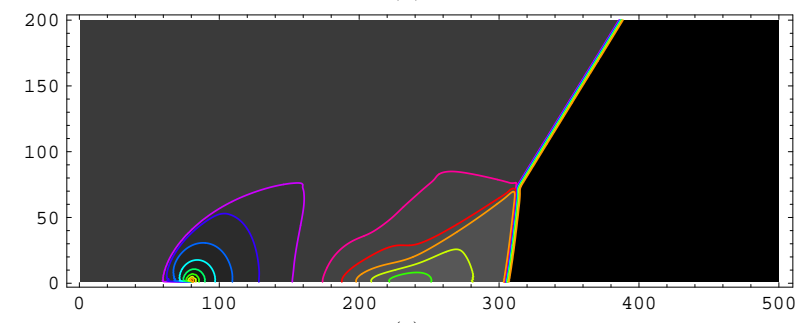

(c)

FIG. 2: Contours of density (a), temperature (b), and $u_{x}$ (c) of the double Mach reflection problem at the time $t=7.5 \times 10^{-3}$. The units of the $x$ - and $y$ - axes are both 0.001 .

sides are:

$$
\begin{gathered}
\left.\left(\rho, u_{x}, u_{y}, T\right)\right|_{x, y, 0}= \\
\begin{cases}\left(\frac{400}{67}, 13.3 \cos 30^{\circ},-13.3 \sin 30^{\circ}, 89.2775\right), & \text { if } y \geq h(x, 0) \\
(2.0,0.0,0.0,0.5), & \text { if } y<h(x, 0)\end{cases}
\end{gathered}
$$

where $h(x, t)=\sqrt{3}(x-80 \Delta x)-40 t$. The reflecting wall lines along the bottom of the problem domain, beginning at $x=0.08$. The shock makes a $60^{\circ}$ angle with the $x$ axis and extends to the top of the problem domain at $y=0.2$. At the top boundary, the physical quantities are assigned the same values as on the left side for $x \leq g(t)$ and are assigned the same values as on the right side for $x>g(t)$, where $g(t)=80 \Delta x+\sqrt{3} / 3(0.2+40 t)$. The computed density, temperature and flow velocity along the $x$-direction are shown in Fig.2, where complex characteristics, such as oblique shocks and triple points, are well captured.

In this model the ratio of specific heat is fixed on an unphysical constant 2. Later, Gan, Xu, Zhang, et al studied a model for flexible specific heat ratio [37]. For higher 
computational efficiency, Chen, Xu, Zhang, et al proposed a model where the number of discrete velocity decreases from 65 to 16 [38]. They simulated the reaction of shock wave on a bubble or ball, etc. In 2010 they present a three-dimensional LB model for high Mach number compressible flows. Figures 3(a) and 3(b) show our successful LB simulations of shock wave reactions on bubble and on ball, respectively, where only the density isosurfaces are shown. In both Figs.(a) and (b), the upper plot shows the initial state, and the lower one shows a snapshot in the shocking procedure. The added additional viscosity makes the scheme more consistent with the physical system and more convenient to satisfy the von Neumann stability condition. Among the discussions on LB model with additional viscosity, the application of flux limiters is also investigated [40]. In the reference with flux limiters [40] Gan, Xu, Zhang, et al also introduced an improved BGK model to break the fixedPrandtl-number barrier. It is meaningful to briefly review the scheme for this improvement.

In the SRT model, both the viscosity and heat conductivity coefficients are proportional to the relaxation time $\tau$. As a result, the Pr is fixed to

$$
\operatorname{Pr}=\frac{c_{p} \mu}{\kappa}=1
$$

The control of Pr may be achieved by modifying the BGK collision term as below:

$$
\frac{\partial f_{k i}}{\partial t}+\mathbf{v}_{k i} \cdot \frac{\partial f_{k i}}{\partial \mathbf{r}}=-\frac{1}{\tau}\left[f_{k i}-(1+\Lambda \tau) f_{k i}^{e q}\right]
$$

where $\Lambda$ takes the following form

$$
\Lambda=A+B\left(\mathbf{v}_{k i}-\mathbf{u}\right)^{2}
$$

Contributions of the new term $\Lambda f_{k i}^{e q}$ in Eq.(22) to the mass, momentum, and energy equations are

$$
\begin{gathered}
\sum_{k i} \Lambda f_{k i}^{e q}=(A+2 B T) \rho=0 \\
\sum_{k i} \Lambda f_{k i}^{e q} v_{k i \alpha}=(A+2 B T) \rho u_{\alpha}=0 \\
\sum_{k i} \frac{1}{2} \Lambda f_{k i}^{e q} v_{k}^{2}=\rho(A+2 B T)\left(T+\frac{u^{2}}{2}\right)+2 \rho T^{2} B=2 \rho T^{2} B .
\end{gathered}
$$

We require that Eq.(22) recovers the Navier-Stokes equations in the following form,

$$
\frac{\partial \rho}{\partial t}+\frac{\partial\left(\rho u_{\alpha}\right)}{\partial r_{\alpha}}=0
$$




$$
\begin{gathered}
\frac{\partial\left(\rho u_{\alpha}\right)}{\partial t}+\frac{\partial\left(\rho u_{\alpha} u_{\beta}+P \delta_{\alpha \beta}\right)}{\partial r_{\beta}}-\frac{\partial}{\partial r_{\beta}}\left[\mu\left(\frac{\partial u_{\beta}}{\partial r_{\alpha}}+\frac{\partial u_{\alpha}}{\partial r_{\beta}}-(\gamma-1) \frac{\partial u_{\gamma}}{\partial r_{\gamma}} \delta_{\alpha \beta}\right)\right]=0 \\
\frac{\partial}{\partial t}\left[\left(E+\frac{\rho u^{2}}{2}\right)\right]+\frac{\partial}{\partial r_{\alpha}}\left[u_{\alpha}\left(E+\frac{\rho u^{2}}{2}+P\right)\right]-\frac{\partial}{\partial r_{\alpha}}\left[\kappa \frac{\partial T}{\partial r_{\alpha}}\right. \\
\left.+\mu u_{\beta}\left(\frac{\partial u_{\beta}}{\partial r_{\alpha}}+\frac{\partial u_{\alpha}}{\partial r_{\beta}}-(\gamma-1) \frac{\partial u_{\gamma}}{\partial r_{\gamma}} \delta_{\alpha \beta}\right)\right]=0
\end{gathered}
$$

where $\mu=\rho T \tau$ is the viscosity, $\kappa$ is the heat conductivity. $\kappa$ is required to be $\kappa=c_{p} \rho T(\tau+q)$, where $c_{p}=\gamma c_{v}=\gamma /(\gamma-1)$ is the specific-heat at constant pressure. It is clear that a new coefficient $q$ is introduced to make the Prandtl number flexible. By using Eqs.(24)-(26) it is easy to find coefficients in Eq.(23) with the following form

$$
A=-2 B T, B=\frac{1}{2 \rho T^{2}} \partial_{\alpha}\left[c_{p} \rho T q \partial_{\alpha} T\right] .
$$

Therefore, the modified BGK collision term changes the heat conductibility in the energy equation from $\kappa=c_{p} \rho T \tau$ to $\kappa=c_{p} \rho T(\tau+q)$. Consequently, the Prandtl number is changed to

$$
\operatorname{Pr}=\frac{\tau}{\tau+q} .
$$

Figure 4 shows a validation example of such a scheme for flexible Prandtl numbers based on the SRT model. The figure shows the comparison of LB results with theoretical solutions for thermal Couette Flows. Fig.(a) is for the temperature profiles in steady state for various Prandtl numbers. Fig.(b) shows the velocity profiles for $\operatorname{Pr}=5.0$ at various times. For more details the readers can refer to Ref. [40]. Such a scheme makes a significant remedy from the side of physical modeling. It is easy to find that such a scheme can also be used to change other transport coefficients such as the viscosity. It is also meaningful to mention that among the moment relations required by each LB model, only for the three, the definitions of density, momentum and energy, the equilibrium distribution function $f_{i}^{e q}$ can be replaced by the distribution function $f_{i}$. If we replace $f_{i}^{e q}$ by $f_{i}$ in RHS of any other required moment relations, the value of RHS will have a deviation from that of the left hand side. This deviation may work as a measure for the deviation of system from its equilibrium. For example, the following $\Delta_{1}$

$$
\Delta_{1}=\sum_{i=0}^{N-1} f_{i} v_{i \alpha} v_{i \beta}^{2}-\sum_{i=0}^{N-1} f_{i}^{e q} v_{i \alpha} v_{i \beta}^{2},
$$

presents a measure for how much the system deviates from its equilibrium for cases without using the constraint $\Delta t=\epsilon$. 


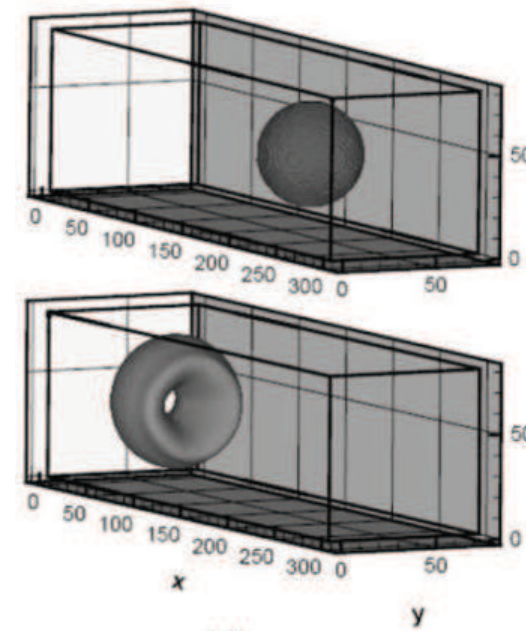

(a)

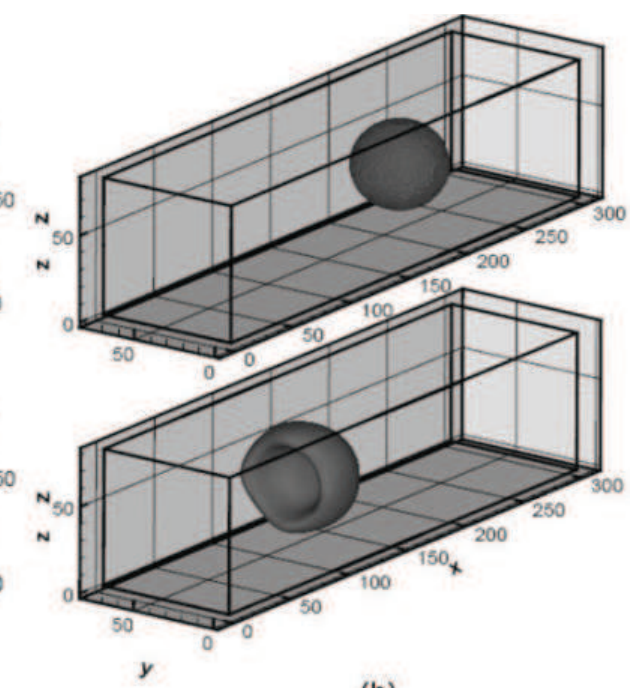

(b)

FIG. 3: Density isosurfaces of shocked bubble (a) and shocked ball (b). In (a) or (b) the upper plot shows the initial state, the bottom one shows the density configuration during the shocking procedure.
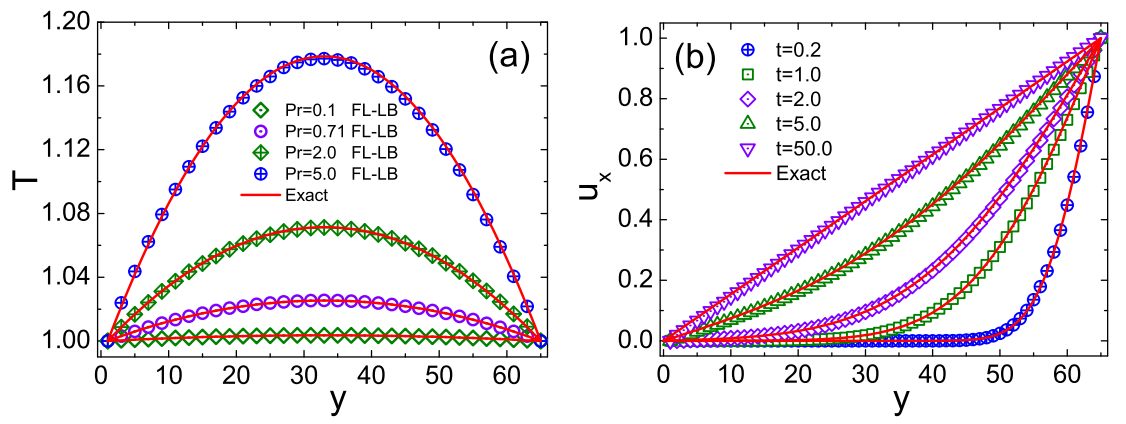

FIG. 4: Comparison of LB results and theoretical solutions for thermal Couette Flows. (a) Temperature profiles in steady state for various Prandtl number. (b) Velocity profiles for $\operatorname{Pr}=5.0$ at various times.

\section{MRT MODEL}

It is known that different motion modes generally approach their equilibria in different velocities. But in the SRT BGK model, the speeds of all discrete distribution functions approaching the equilibria are determined by a single relaxation time $\tau$. That means $\tau$ is an averaged relaxation time of all kinds of motion modes. The best merit of this treatment is that it is simple and keeps the most fundamental conservation laws. This BGK model has been successfully applied in various fields. But with increasing the Mach number and 
Reynolds number, the problem of numerical instability becomes more serious. At the same time, the Prandtl number effect is a key issue in many fluidic systems. Facing with all these requirements and challenges, people began to reevaluate this simple averaging treatment.

The numerical instability of LB simulation is still a difficult problem nowadays. Roughly speaking, the possible reasons come from two sides, the physical modeling and the discretization scheme. It has been indicated that untying the motion modes which should be independent is helpful for improving the numerical stability [46 50]. Succi, et al [46], Luo, et al [48 -50] and many others have made significant contributions in constructing MRT LB models. Those MRT models are mainly within the framework of the standard LB model and work for isothermal systems with low Mach number. In recent years our group proposed two schemes to compose MRT model for high speed compressible flows. These schemes are for the framework of the FD-LB model. The finished works focus still on the two-dimensional cases.

In the MRT LB formulation, the collision step is first calculated in the kinetic moment space spanned by a suitable set of $N$ kinetic moments of the distribution function $f_{i}$. Then, the propagation step is performed back in the discrete velocity space spanned by the $N$ discrete velocities $\mathbf{v}_{i}$. In contrast to the $\mathrm{SRT}$ model, the MRT version caters for more adjustable parameters and degrees of freedom. The relaxation rates of the various kinetic moments due to particle collisions may be adjusted independently. The MRT LB equation has the following form,

$$
\frac{\partial f_{i}}{\partial t}+v_{i \alpha} \frac{\partial f_{i}}{\partial x_{\alpha}}=-\mathbf{S}_{i k}\left[f_{k}-f_{k}^{e q}\right],
$$

where $\mathbf{S}$ is the collision matrix. The equation reduces to the usual lattice BGK equation if all the relaxation parameters are set to be a single relaxation time $\tau$, namely $\mathbf{S}=\frac{1}{\tau} \mathbf{I}$, where $\mathbf{I}$ is the identity matrix. The discrete distribution functions $f_{i}$ and $f_{i}^{e q}$ can be rewritten as the following matrixes:

$$
\begin{aligned}
\mathbf{f} & =\left(f_{1}, f_{2}, \cdots, f_{N}\right)^{T}, \\
\mathbf{f}^{e q} & =\left(f_{1}^{e q}, f_{2}^{e q}, \cdots, f_{N}^{e q}\right)^{T},
\end{aligned}
$$

where $T$ is the transpose operator. Given a set of discrete velocities $\mathbf{v}_{i}$ and corresponding distribution functions $f_{i}$, we can get a velocity space $S^{V}$ spanned by discrete velocities $\mathbf{v}_{i}$ and a moment space $S^{M}$ spanned by moments of particle distribution function $f_{i}$. The moments of particle distribution function reads $\hat{\mathbf{f}}=\left(\hat{f}_{1}, \hat{f}_{2}, \cdots, \hat{f}_{N}\right)^{T}$, where $\hat{f}_{i}=m_{i j} f_{j}$, 
$m_{i j}$ is an element of the matrix $\mathbf{M}$ and is a polynomial of discrete velocities. Obviously, the moments are simply linear combination of distribution functions $f_{i}$, and the mapping between moment space and velocity space is defined by the linear transformation M, i.e., $\hat{\mathbf{f}}=\mathbf{M f}, \mathbf{f}=\mathbf{M}^{-1} \hat{\mathbf{f}}$, where $\mathbf{M}=\left(m_{1}, m_{2}, \cdots, m_{N}\right)^{T}, m_{i}=\left(m_{i 1}, m_{i 2}, \cdots, m_{i N}\right)$.

Since the collision step is first calculated in the moment space and then mapped back to the velocity space. So, the MRT LB equation can be described as

$$
\frac{\partial f_{i}}{\partial t}+v_{i \alpha} \frac{\partial f_{i}}{\partial x_{\alpha}}=-\mathbf{M}_{i l}^{-1} \hat{\mathbf{S}}_{l k}\left(\hat{f}_{k}-\hat{f}_{k}^{e q}\right)
$$

where $\hat{\mathbf{S}}=\mathbf{M S M}^{-1}=\operatorname{diag}\left(s_{1}, s_{2}, \cdots, s_{N}\right)$ is a diagonal relaxation matrix. $\hat{f}_{i}^{e q}$ is the equilibrium value of the moment $\hat{f}_{i}$. The moments can be divided into two groups. The first group consists of the moments locally conserved in the collision process, i.e. $\hat{f}_{i}=\hat{f}_{i}^{e q}$. The second group consists of the moments not conserved, i.e. $\hat{f}_{i} \neq \hat{f}_{i}^{e q}$. The equilibrium $\hat{f}_{i}^{e q}$ is a function of conserved moments. It is clear that the first group includes the density, the momentum and the energy.

\section{A. MRT model based on group representation theory}

Now we briefly review the first MRT LB model proposed in our group [41]. Our first MRT model is developed from the SRT version by Kataoka and Tsutahara [25]. The DVM can be expressed as:

$$
\left(v_{i x}, v_{i y}\right)=\left\{\begin{array}{lc}
\text { cyc }:( \pm 1,0), & \text { for } 1 \leq i \leq 4 \\
\text { cyc }:( \pm 6,0), & \text { for } 5 \leq i \leq 8 \\
\sqrt{2}( \pm 1, \pm 1), & \text { for } 9 \leq i \leq 12 \\
\frac{3}{\sqrt{2}}( \pm 1, \pm 1), & \text { for } 13 \leq i \leq 16
\end{array}\right.
$$

where cyc indicates the cyclic permutation. (see Fig. 5) 


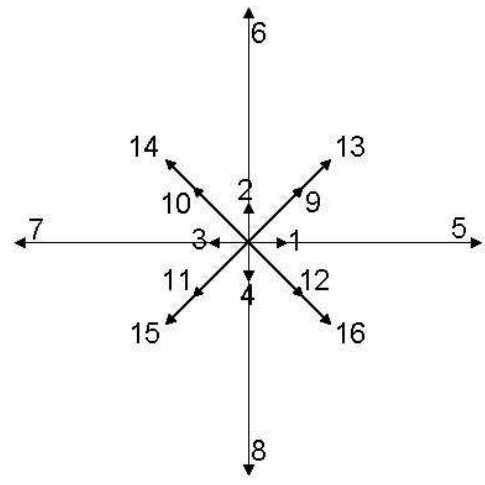

FIG. 5: Distribution of $\mathbf{v}_{i \alpha}$ for the discrete velocity model.

\section{Construction of transformation matrix $\mathbf{M}$}

The transformation matrix $\mathbf{M}$ is constructed according to the irreducible representations of $\mathrm{SO}(2)$ group:

$$
\begin{aligned}
& 1, \\
& \cos \theta, \sin \theta, \\
& \sin ^{2} \theta+\cos ^{2} \theta, \cos 2 \theta, \sin 2 \theta, \\
& \cos \theta\left(\sin ^{2} \theta+\cos ^{2} \theta\right), \sin \theta\left(\sin ^{2} \theta+\cos ^{2} \theta\right), \cos 3 \theta, \sin 3 \theta, \\
& \left(\sin ^{2} \theta+\cos ^{2} \theta\right)^{2}, \cos 4 \theta, \cos 2 \theta\left(\sin ^{2} \theta+\cos ^{2} \theta\right), \\
& \sin 2 \theta\left(\sin ^{2} \theta+\cos ^{2} \theta\right), \\
& \cos 3 \theta\left(\sin ^{2} \theta+\cos ^{2} \theta\right), \sin 3 \theta\left(\sin ^{2} \theta+\cos ^{2} \theta\right), \cdots
\end{aligned}
$$

Let $v_{i x}$ and $v_{i y}$ play the roles of $\cos \theta$ and $\sin \theta$, respectively. Then we define $m_{1 i}=1$, $m_{2 i}=v_{i x}, m_{3 i}=v_{i y}, m_{4 i}=\left(v_{i x}^{2}+v_{i y}^{2}\right) / 2, m_{5 i}=v_{i x}^{2}-v_{i y}^{2}, m_{6 i}=v_{i x} v_{i y}, m_{7 i}=v_{i x}\left(v_{i x}^{2}+v_{i y}^{2}\right) / 2$, $m_{8 i}=v_{i y}\left(v_{i x}^{2}+v_{i y}^{2}\right) / 2, m_{9 i}=v_{i x}\left(v_{i x}^{2}-3 v_{i y}^{2}\right), m_{10 i}=v_{i y}\left(3 v_{i x}^{2}-v_{i y}^{2}\right), m_{11 i}=\left(v_{i x}^{2}+v_{i y}^{2}\right)^{2} / 4$, $m_{12 i}=v_{i x}^{4}-6 v_{i x}^{2} v_{i y}^{2}+v_{i y}^{4}, m_{13 i}=\left(v_{i x}^{2}+v_{i y}^{2}\right)\left(v_{i x}^{2}-v_{i y}^{2}\right), m_{14 i}=\left(v_{i x}^{2}+v_{i y}^{2}\right) v_{i x} v_{i y}, m_{15 i}=$ $v_{i x}\left(v_{i x}^{2}-3 v_{i y}^{2}\right)\left(v_{i x}^{2}+v_{i y}^{2}\right), m_{16 i}=v_{i y}\left(3 v_{i x}^{2}-v_{i y}^{2}\right)\left(v_{i x}^{2}+v_{i y}^{2}\right)$, where $i=1, \cdots, 16$.

For two-dimensional compressible models, we have four conserved moments, density $\hat{f}_{1}=$ $\rho=\sum f_{i} m_{1 i}$, momenta $\hat{f}_{2}=j_{x}=\rho u_{x}=\sum f_{i} m_{2 i}$ and $\hat{f}_{3}=j_{y}=\rho u_{y}=\sum f_{i} m_{3 i}$, and energy $\hat{f}_{4}=e=\sum f_{i} m_{4 i}$. To be consistent with the idiomatic expression of energy, in the definitions of $m_{4 i}, m_{7 i}$ and $m_{8 i}$, a coefficient $1 / 2$ is used. Similarly, a coefficient $1 / 4$ is used in the definition of $m_{11 i}$. The components of transformation matrix $\mathbf{M}$ are shown in table I. 
TABLE I: Transformation matrix of MRT-LB for compressible fluids.

\begin{tabular}{ccccccccccccccccc}
\hline \hline$i$ & $m_{1 i}$ & $m_{2 i}$ & $m_{3 i}$ & $m_{4 i}$ & $m_{5 i}$ & $m_{6 i}$ & $m_{7 i}$ & $m_{8 i}$ & $m_{9 i}$ & $m_{10 i}$ & $m_{11 i}$ & $m_{12 i}$ & $m_{13 i}$ & $m_{14 i}$ & $m_{15 i}$ & $m_{16 i}$ \\
\hline 1 & 1 & 1 & 0 & $\frac{1}{2}$ & 1 & 0 & $\frac{1}{2}$ & 0 & 1 & 0 & $\frac{1}{4}$ & 1 & 1 & 0 & 1 & 0 \\
2 & 1 & 0 & 1 & $\frac{1}{2}$ & -1 & 0 & 0 & $\frac{1}{2}$ & 0 & -1 & $\frac{1}{4}$ & 1 & -1 & 0 & 0 & -1 \\
3 & 1 & -1 & 0 & $\frac{1}{2}$ & 1 & 0 & $-\frac{1}{2}$ & 0 & -1 & 0 & $\frac{1}{4}$ & 1 & 1 & 0 & -1 & 0 \\
4 & 1 & 0 & -1 & $\frac{1}{2}$ & -1 & 0 & 0 & $-\frac{1}{2}$ & 0 & 1 & $\frac{1}{4}$ & 1 & -1 & 0 & 0 & 1 \\
5 & 1 & 6 & 0 & 18 & 36 & 0 & 108 & 0 & 216 & 0 & 324 & 1296 & 1296 & 0 & 7776 & 0 \\
6 & 1 & 0 & 6 & 18 & -36 & 0 & 0 & 108 & 0 & -216 & 324 & 1296 & -1296 & 0 & 0 & -7776 \\
7 & 1 & -6 & 0 & 18 & 36 & 0 & -108 & 0 & -216 & 0 & 324 & 1296 & 1296 & 0 & -7776 & 0 \\
8 & 1 & 0 & -6 & 18 & -36 & 0 & 0 & -108 & 0 & 216 & 324 & 1296 & -1296 & 0 & 0 & 7776 \\
9 & 1 & $\sqrt{2}$ & $\sqrt{2}$ & 2 & 0 & 2 & $2 \sqrt{2}$ & $2 \sqrt{2}$ & $-4 \sqrt{2}$ & $4 \sqrt{2}$ & 4 & -16 & 0 & 8 & $-16 \sqrt{2}$ & $16 \sqrt{2}$ \\
10 & 1 & $-\sqrt{2}$ & $\sqrt{2}$ & 2 & 0 & -2 & $-2 \sqrt{2}$ & $2 \sqrt{2}$ & $4 \sqrt{2}$ & $4 \sqrt{2}$ & 4 & -16 & 0 & -8 & $16 \sqrt{2}$ & $16 \sqrt{2}$ \\
11 & 1 & $-\sqrt{2}$ & $-\sqrt{2}$ & 2 & 0 & 2 & $-2 \sqrt{2}$ & $-2 \sqrt{2}$ & $4 \sqrt{2}$ & $-4 \sqrt{2}$ & 4 & -16 & 0 & 8 & $16 \sqrt{2}$ & $-16 \sqrt{2}$ \\
12 & 1 & $\sqrt{2}$ & $-\sqrt{2}$ & 2 & 0 & -2 & $2 \sqrt{2}$ & $-2 \sqrt{2}$ & $-4 \sqrt{2}$ & $-4 \sqrt{2}$ & 4 & -16 & 0 & -8 & $-16 \sqrt{2}$ & $-16 \sqrt{2}$ \\
13 & 1 & $\frac{3}{\sqrt{2}}$ & $\frac{3}{\sqrt{2}}$ & $\frac{9}{2}$ & 0 & $\frac{9}{2}$ & $\frac{27}{2 \sqrt{2}}$ & $\frac{27}{2 \sqrt{2}}$ & $-\frac{27}{\sqrt{2}}$ & $\frac{27}{\sqrt{2}}$ & $\frac{81}{4}$ & -81 & 0 & $\frac{81}{2}$ & $-\frac{243}{\sqrt{2}}$ & $\frac{243}{\sqrt{2}}$ \\
14 & 1 & $-\frac{3}{\sqrt{2}}$ & $\frac{3}{\sqrt{2}}$ & $\frac{9}{2}$ & 0 & $-\frac{9}{2}$ & $-\frac{27}{2 \sqrt{2}}$ & $\frac{27}{2 \sqrt{2}}$ & $\frac{27}{\sqrt{2}}$ & $\frac{27}{\sqrt{2}}$ & $\frac{81}{4}$ & -81 & 0 & $-\frac{81}{2}$ & $\frac{243}{\sqrt{2}}$ & $\frac{243}{\sqrt{2}}$ \\
15 & 1 & $-\frac{3}{\sqrt{2}}-\frac{3}{\sqrt{2}}$ & $\frac{9}{2}$ & 0 & $\frac{9}{2}$ & $-\frac{27}{2 \sqrt{2}}$ & $-\frac{27}{2 \sqrt{2}}$ & $\frac{27}{\sqrt{2}}$ & $-\frac{27}{\sqrt{2}}$ & $\frac{81}{4}$ & -81 & 0 & $\frac{81}{2}$ & $\frac{243}{\sqrt{2}}$ & $-\frac{243}{\sqrt{2}}$ \\
16 & 1 & $\frac{3}{\sqrt{2}}$ & $-\frac{3}{\sqrt{2}}$ & $\frac{9}{2}$ & 0 & $-\frac{9}{2}$ & $\frac{27}{2 \sqrt{2}}$ & $-\frac{27}{2 \sqrt{2}}$ & $-\frac{27}{\sqrt{2}}$ & $-\frac{27}{\sqrt{2}}$ & $\frac{81}{4}$ & -81 & 0 & $-\frac{81}{2}$ & $-\frac{243}{\sqrt{2}}$ & $-\frac{243}{\sqrt{2}}$ \\
\hline \hline
\end{tabular}

\section{Determination of $\hat{f}_{i}^{e q}$}

The second group components of $\hat{f}_{i}^{e q}$ are chosen in such a way that in the continuum limit the MRT LB model recovers the Navier-Stokes equations. To that end, we perform the Chapman-Enskog expansion on the two sides of Eq.(33). We use the following multiscale expansions:

$$
\begin{gathered}
f_{i}=f_{i}^{(0)}+f_{i}^{(1)}+f_{i}^{(2)}, \\
\frac{\partial}{\partial t}=\frac{\partial}{\partial t_{1}}+\frac{\partial}{\partial t_{2}}, \\
\frac{\partial}{\partial x}=\frac{\partial}{\partial x_{1}},
\end{gathered}
$$

where $f_{i}^{(0)}$ is the zeroth order, $f_{i}^{(1)}, \frac{\partial}{\partial t_{1}}$ and $\frac{\partial}{\partial x_{1}}$ are the first order, $f_{i}^{(2)}$ and $\frac{\partial}{\partial t_{2}}$ are the second order terms of the Knudsen number $\epsilon$. Equating the coefficients of the zeroth, the first, and 
the second order terms in $\epsilon$ gives

$$
\begin{gathered}
f_{i}^{(0)}=f_{i}^{e q} \\
\left(\frac{\partial}{\partial t_{1}}+v_{i \alpha} \frac{\partial}{\partial x_{1 \alpha}}\right) f_{i}^{(0)}=-\mathbf{S}_{i l} f_{l}^{(1)} \\
\frac{\partial}{\partial t_{2}} f_{i}^{(0)}+\left(\frac{\partial}{\partial t_{1}}+v_{i \alpha} \frac{\partial}{\partial x_{1 \alpha}}\right) f_{i}^{(1)}=-\mathbf{S}_{i l} f_{l}^{(2)} .
\end{gathered}
$$

In the moment space they are

$$
\begin{gathered}
\hat{f}_{i}^{(0)}=\hat{f}_{i}^{e q}, \\
\left(\frac{\partial}{\partial t_{1}}+\hat{\mathbf{E}}_{\alpha} \frac{\partial}{\partial x_{1 \alpha}}\right) \hat{f}_{i}^{(0)}=-\hat{\mathbf{S}}_{i l} \hat{f}_{l}^{(1)}, \\
\frac{\partial}{\partial t_{2}} \hat{f}_{i}^{(0)}+\left(\frac{\partial}{\partial t_{1}}+\hat{\mathbf{E}}_{\alpha} \frac{\partial}{\partial x_{1 \alpha}}\right) \hat{f}_{i}^{(1)}=-\hat{\mathbf{S}}_{i l} \hat{f}_{l}^{(2)},
\end{gathered}
$$

where $\hat{\mathbf{E}}_{\alpha}=\mathbf{M}\left(v_{i \alpha} \mathbf{I}\right) \mathbf{M}^{-1}$.

The equilibria of the moments in the moment space read $\hat{\mathbf{f}}^{e q}=$ $\left(\rho, j_{x}, j_{y}, e, \hat{f}_{5}^{e q}, \hat{f}_{6}^{e q}, \cdots, \hat{f}_{16}^{e q}\right)^{T}$. The first and second order deviations from equilibria are defined as : $\hat{\mathbf{f}}^{(1)}=\left(0,0,0,0, \hat{f}_{5}^{(1)}, \hat{f}_{6}^{(1)}, \cdots, \hat{f}_{16}^{(1)}\right)^{T}$ and $\hat{\mathbf{f}}^{(2)}=\left(0,0,0,0, \hat{f}_{5}^{(2)}, \hat{f}_{6}^{(2)}, \cdots, \hat{f}_{16}^{(2)}\right)^{T}$, respectively. Via some algebraic treatments, we obtain

$$
\begin{gathered}
\frac{\partial \rho}{\partial t}+\frac{\partial j_{x}}{\partial x}+\frac{\partial j_{y}}{\partial y}=0 \\
\frac{\partial j_{x}}{\partial t}+\frac{\partial}{\partial x}\left(e+\frac{1}{2} \hat{f}_{5}^{e q}\right)+\frac{\partial}{\partial y} \hat{f}_{6}^{e q}=-\frac{1}{2} \frac{\partial}{\partial x} \hat{f}_{5}^{(1)}-\frac{\partial}{\partial y} \hat{f}_{6}^{(1)}, \\
\frac{\partial j_{y}}{\partial t}+\frac{\partial}{\partial x} \hat{f}_{6}^{e q}+\frac{\partial}{\partial y}\left(e-\frac{1}{2} \hat{f}_{5}^{e q}\right)=-\frac{\partial}{\partial x} \hat{f}_{6}^{(1)}+\frac{1}{2} \frac{\partial}{\partial y} \hat{f}_{5}^{(1)}, \\
\frac{\partial e}{\partial t}+\frac{\partial}{\partial x} \hat{f}_{7}^{e q}+\frac{\partial}{\partial y} \hat{f}_{8}^{e q}=-\frac{\partial}{\partial x} \hat{f}_{7}^{(1)}-\frac{\partial}{\partial y} \hat{f}_{8}^{(1)} .
\end{gathered}
$$

If choose $\hat{f}_{5}^{e q}=\left(j_{x}^{2}-j_{y}^{2}\right) / \rho, \hat{f}_{6}^{e q}=j_{x} j_{y} / \rho, \hat{f}_{7}^{e q}=(e+P) j_{x} / \rho, \hat{f}_{8}^{e q}=(e+P) j_{y} / \rho, \hat{f}_{9}^{e q}=$ $\left(j_{x}^{2}-3 j_{y}^{2}\right) j_{x} / \rho^{2}, \hat{f}_{10}^{e q}=\left(3 j_{x}^{2}-j_{y}^{2}\right) j_{y} / \rho^{2}, \hat{f}_{11}^{e q}=2 e^{2} / \rho-\left(j_{x}^{2}+j_{y}^{2}\right)^{2} / 4 \rho^{3}, \hat{f}_{13}^{e q}=\left(6 \rho e-2 j_{x}^{2}-\right.$ $\left.2 j_{y}^{2}\right)\left(j_{x}^{2}-j_{y}^{2}\right) / \rho^{3}, \hat{f}_{14}^{e q}=\left(6 \rho e-2 j_{x}^{2}-2 j_{y}^{2}\right) j_{x} j_{y} / \rho^{3}, \hat{f}_{12}^{e q}=\hat{f}_{15}^{e q}=\hat{f}_{16}^{e q}=0$, the MRT LB model recovers the following Navier-Stokes equations:

$$
\begin{gathered}
\frac{\partial \rho}{\partial t}+\frac{\partial j_{x}}{\partial x}+\frac{\partial j_{y}}{\partial y}=0 \\
\frac{\partial j_{x}}{\partial t}+\frac{\partial}{\partial x}\left(j_{x}^{2} / \rho\right)+\frac{\partial}{\partial y}\left(j_{x} j_{y} / \rho\right)=-\frac{\partial P}{\partial x}+\frac{\partial}{\partial x}\left[\mu_{s}\left(\frac{\partial u_{x}}{\partial x}-\frac{\partial u_{y}}{\partial y}\right)\right]+\frac{\partial}{\partial y}\left[\mu_{v}\left(\frac{\partial u_{y}}{\partial x}+\frac{\partial u_{x}}{\partial y}\right)\right] \\
\frac{\partial j_{y}}{\partial t}+\frac{\partial}{\partial x}\left(j_{x} j_{y} / \rho\right)+\frac{\partial}{\partial y}\left(j_{y}^{2} / \rho\right)=-\frac{\partial P}{\partial y}+\frac{\partial}{\partial x}\left[\mu_{v}\left(\frac{\partial u_{y}}{\partial x}+\frac{\partial u_{x}}{\partial y}\right)\right]-\frac{\partial}{\partial y}\left[\mu_{s}\left(\frac{\partial u_{x}}{\partial x}-\frac{\partial u_{y}}{\partial y}\right)\right]
\end{gathered}
$$




$$
\begin{aligned}
& \frac{\partial e}{\partial t}+\frac{\partial}{\partial x}\left[(e+P) j_{x} / \rho\right]+\frac{\partial}{\partial y}\left[(e+P) j_{y} / \rho\right] \\
= & \frac{\partial}{\partial x}\left[\lambda_{1} \frac{\partial T}{\partial x}+\frac{\lambda_{1}}{2}\left(u_{y} \frac{\partial u_{y}}{\partial x}+u_{x} \frac{\partial u_{x}}{\partial x}-u_{x} \frac{\partial u_{y}}{\partial y}+u_{y} \frac{\partial u_{x}}{\partial y}\right)\right] \\
& +\frac{\partial}{\partial y}\left[\lambda_{2} \frac{\partial T}{\partial y}+\frac{\lambda_{2}}{2}\left(u_{x} \frac{\partial u_{x}}{\partial y}-u_{y} \frac{\partial u_{x}}{\partial x}+u_{x} \frac{\partial u_{y}}{\partial x}+u_{y} \frac{\partial u_{y}}{\partial y}\right)\right],
\end{aligned}
$$

where $\mu_{s}=\rho R T / s_{5}, \mu_{v}=\rho R T / s_{6}, \lambda_{1}=2 \rho R T / s_{7}, \lambda_{2}=2 \rho R T / s_{8}$. It is noted that the definitions of $\hat{f}_{12}^{e q}, \hat{f}_{15}^{e q}, \hat{f}_{16}^{e q}$ have no effect on the recovered macroscopic equations. When $\mu_{s}=\mu_{v}=\mu, \lambda_{1}=\lambda_{2}=\lambda$, the above Navier-Stokes equations reduce to

$$
\begin{gathered}
\frac{\partial \rho}{\partial t}+\frac{\partial j_{\alpha}}{\partial x_{\alpha}}=0 \\
\frac{\partial j_{\alpha}}{\partial t}+\frac{\partial\left(j_{\alpha} j_{\beta} / \rho\right)}{\partial x_{\beta}}=-\frac{\partial P}{\partial x_{\alpha}}+\frac{\partial}{\partial x_{\beta}}\left[\mu\left(\frac{\partial u_{\alpha}}{\partial x_{\beta}}+\frac{\partial u_{\beta}}{\partial x_{\alpha}}-\frac{\partial u_{\chi}}{\partial x_{\chi}} \delta_{\alpha \beta}\right)\right] \\
\frac{\partial e}{\partial t}+\frac{\partial}{\partial x_{\alpha}}\left[(e+P) j_{\alpha} / \rho\right]=\frac{\partial}{\partial x_{\alpha}}\left[\lambda \frac{\partial T}{\partial x_{\alpha}}+\frac{\lambda}{2} u_{\beta}\left(\frac{\partial u_{\alpha}}{\partial x_{\beta}}+\frac{\partial u_{\beta}}{\partial x_{\alpha}}-\frac{\partial u_{\chi}}{\partial x_{\chi}} \delta_{\alpha \beta}\right)\right] .
\end{gathered}
$$

In Fig.6(a) we show an example of stability comparison for the new MRT model and its SRT version. The abscissa is for $k d x$, and the vertical axis is for $|\omega|_{\max }$ which is the largest eigenvalue of coefficient matrix $G_{i j}$. The macroscopic values in stability analysis are chosen as follows: $\left(\rho, u_{x}, u_{y}, T\right)=(2.0,10.0,0.0,2.0)$. The relaxation time in SRT is $\tau=10^{-5}$, while the collision parameters in MRT are $s_{5}=6500, s_{7}=s_{8}=9 \times 10^{4}, s_{9}=8 \times 10^{4}, s_{13}=7 \times 10^{4}$, $s_{14}=8 \times 10^{3}, s_{15}=2.5 \times 10^{4}$, the others are $10^{5}$. In this case, the MRT scheme is stable, while the SRT version is not. It is clear that, by choosing appropriate collision parameters, the stability of MRT can be much better than the SRT.

Figure 6(b) shows the comparison of MRT LB results and exact ones for the well-known Colella explosion wave problem. For the problem, the initial condition is

$$
\left\{\begin{array}{c}
\left.\left(\rho, u_{x}, u_{y}, T\right)\right|_{L}=(1.0,0.0,0.0,1000.0), x \leq 0 \\
\left.\left(\rho, u_{x}, u_{y}, T\right)\right|_{R}=(1.0,0.0,0.0,0.01), \quad x>0
\end{array}\right.
$$

This is a strong temperature discontinuity problem that can be used to study the robustness and precision of numerical methods. Figure 6(b) gives density, pressure, velocity and temperature results at $t=0.1$. Symbols are for simulation results. Here, the parameters are $s_{7}=s_{8}=5 \times 10^{4}, s_{11}=s_{13}=5 \times 10^{5}$, other values of $s$ still adopt $10^{5}$. The success of the simulation shows that the MRT model is applicable to simulate strong temperature discontinuity problem, and confirms the robustness and precision of the model. 


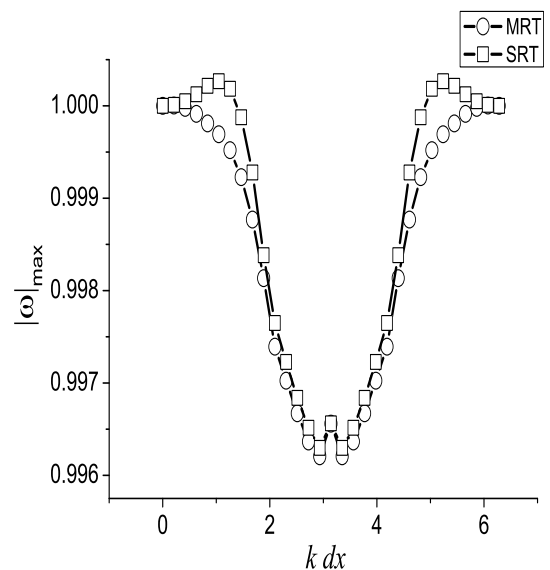

(a)
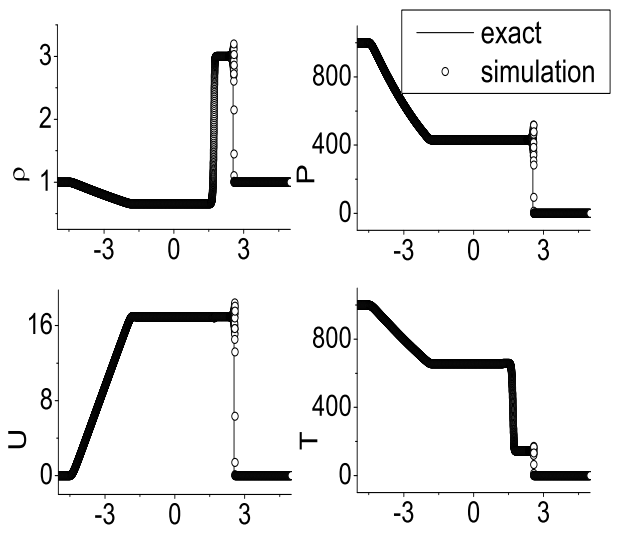

(b)

FIG. 6: (a) Stability comparison for the new MRT model and its SRT version. (b) The MRT simulation results and exact solutions for the Colella explosion wave at time $t=0.1$.

Two points should be commented here. The first is that the better stability is not the only or most important advantage of MRT over SRT. From the view of physical modeling, the SRT is only a special case of the MRT. The second is that the above MRT LB model works well for shocked compressible fluids where the shocking procedure is much faster than the transportation processes. To work also well for more general cases, the collision operators of the moments related to the energy flux should be modified as below [45],

$\hat{\mathbf{S}}_{77}\left(\hat{f}_{7}-\hat{f}_{7}^{e q}\right) \Rightarrow \hat{\mathbf{S}}_{77}\left(\hat{f}_{7}-\hat{f}_{7}^{e q}\right)+\left(s_{7} / s_{5}-1\right) \rho T u_{x}\left(\frac{\partial u_{x}}{\partial x}-\frac{\partial u_{y}}{\partial y}\right)+\left(s_{7} / s_{6}-1\right) \rho T u_{y}\left(\frac{\partial u_{y}}{\partial x}+\frac{\partial u_{x}}{\partial y}\right)$,

$\hat{\mathbf{S}}_{88}\left(\hat{f}_{8}-\hat{f}_{8}^{e q}\right) \Rightarrow \hat{\mathbf{S}}_{88}\left(\hat{f}_{8}-\hat{f}_{8}^{e q}\right)+\left(s_{8} / s_{6}-1\right) \rho T u_{x}\left(\frac{\partial u_{y}}{\partial x}+\frac{\partial u_{x}}{\partial y}\right)-\left(s_{8} / s_{5}-1\right) \rho T u_{y}\left(\frac{\partial u_{x}}{\partial x}-\frac{\partial u_{y}}{\partial y}\right)$.

After the modification the coefficients of viscosity in energy equation (41d) are consistent with those in momentum equations (41b)-(41c).

\section{B. MRT model based on moment relations}

In the original KT model, besides Eqs. (3)-(7), the local equilibrium distribution function $f_{i}^{e q}$ is required to satisfy the following two additional moment relations:

$$
\rho\left[R T\left(u_{\alpha} \delta_{\beta \chi}+u_{\beta} \delta_{\alpha \chi}+u_{\chi} \delta_{\alpha \beta}\right)+u_{\alpha} u_{\beta} u_{\chi}\right]=\sum f_{i}^{e q} v_{i \alpha} v_{i \beta} v_{i \chi},
$$




$$
\rho\left\{(b+2) R^{2} T^{2} \delta_{\alpha \beta}+\left[(b+4) u_{\alpha} u_{\beta}+u_{\chi}^{2} \delta_{\alpha \beta}\right] R T+u_{\chi}^{2} u_{\alpha} u_{\beta}\right\}=\sum f_{i}^{e q}\left(v_{i \chi}^{2}+\eta_{i}^{2}\right) v_{i \alpha} v_{i \beta}
$$

The local equilibrium distribution function $f_{i}^{e q}$ is calculated via the following polynomial:

$$
\begin{aligned}
f_{i}^{e q}= & \rho\left[a_{0 i}+a_{1 i} T+a_{2 i} T^{2}+\left(a_{3 i}+a_{4 i} T\right) u_{\alpha}^{2}+a_{5 i} u_{\alpha}^{2} u_{\beta}^{2}\right. \\
& +\left(b_{0 i}+b_{1 i} T+b_{2 i} u_{\alpha}^{2}\right) u_{\beta} v_{i \beta}+\left(d_{0 i}+d_{1 i} T+d_{2 i} u_{\alpha}^{2}\right) u_{\beta} v_{i \beta} u_{\chi} v_{i \chi} \\
& \left.+e_{i} u_{\alpha} v_{i \alpha} u_{\beta} v_{i \beta} u_{\chi} v_{i \chi}\right]
\end{aligned}
$$

which is of the flow velocity up to the third order. The coefficients $a_{0 i}, \ldots, e_{i}(i=1, \ldots, 16$ ) in the distribution function $f_{i}^{e q}$ are referred to the original publication [25].

\section{Construction of transformation matrix $\mathbf{M}$}

In this MRT model the moments are chosen according to the seven required moment

relations [42, 43]. The RHS of the seven equations indicate seven monomials: $1, v_{i \alpha}, v_{i \alpha}^{2}+\eta_{i}^{2}$, $v_{i \alpha} v_{i \beta},\left(v_{i \beta}^{2}+\eta_{i}^{2}\right) v_{i \alpha}, v_{i \alpha} v_{i \beta} v_{i \chi},\left(v_{i \chi}^{2}+\eta_{i}^{2}\right) v_{i \alpha} v_{i \beta}$. Three possibilities arise from the monomial $v_{i \alpha} v_{i \beta}$ : (a) $\alpha=\beta=x, v_{i \alpha} v_{i \beta}=v_{i x}^{2}$, (b) $\alpha=\beta=y, v_{i \alpha} v_{i \beta}=v_{i y}^{2}$, (c) $\alpha=x, \beta=y$, $v_{i \alpha} v_{i \beta}=v_{i x} v_{i y} \cdot$ " $(\mathrm{a})+(\mathrm{b})$ " gives $\left(v_{i x}^{2}+v_{i y}^{2}\right)$, “( $\left.\mathrm{a}\right)-(\mathrm{b})$ " gives $\left(v_{i x}^{2}-v_{i y}^{2}\right)$. Through such a simple combination of these monomials, we can compose the transformation matrix $\mathbf{M}$ as below: $m_{1 i}=1, m_{2 i}=v_{i x}, m_{3 i}=v_{i y}, m_{4 i}=v_{i x}^{2}+v_{i y}^{2}+\eta_{i}^{2}, m_{5 i}=v_{i x}^{2}+v_{i y}^{2}, m_{6 i}=v_{i x}^{2}-v_{i y}^{2}$, $m_{7 i}=v_{i x} v_{i y}, m_{8 i}=v_{i x}\left(v_{i x}^{2}+v_{i y}^{2}+\eta_{i}^{2}\right), m_{9 i}=v_{i y}\left(v_{i x}^{2}+v_{i y}^{2}+\eta_{i}^{2}\right), m_{10 i}=v_{i x}\left(v_{i x}^{2}+v_{i y}^{2}\right)$, $m_{11 i}=v_{i y}\left(v_{i x}^{2}+v_{i y}^{2}\right), m_{12 i}=v_{i x}\left(v_{i x}^{2}-v_{i y}^{2}\right), m_{13 i}=v_{i y}\left(v_{i x}^{2}-v_{i y}^{2}\right), m_{14 i}=\left(v_{i x}^{2}+v_{i y}^{2}\right)\left(v_{i x}^{2}+v_{i y}^{2}+\eta_{i}^{2}\right)$, $m_{15 i}=v_{i x} v_{i y}\left(v_{i x}^{2}+v_{i y}^{2}+\eta_{i}^{2}\right), m_{16 i}=\left(v_{i x}^{2}-v_{i y}^{2}\right)\left(v_{i x}^{2}+v_{i y}^{2}+\eta_{i}^{2}\right)$, where $i=1, \cdots, 16$. The components of transformation matrix $\mathbf{M}$ are shown in table II.

It should be pointed out that, different from the other MRT models for isothermal fluids, the transformation matrix $\mathbf{M}$ should not be based upon a Gram-Schmidt orthogonalization procedure.

\section{Determination of $\hat{f}_{i}^{e q}$}

The procedure of determining $\hat{\mathbf{f}}^{e q}$ is similar to that for the first MRT LB model in this paper. But the results are significantly different. Our choice for this model is as below: 
TABLE II: Transformation matrix of MRT-LB model for compressible flows with flexible specificheat ratio.

\begin{tabular}{ccccccccccccccccc}
\hline \hline$i$ & $m_{1 i}$ & $m_{2 i}$ & $m_{3 i}$ & $m_{4 i}$ & $m_{5 i}$ & $m_{6 i}$ & $m_{7 i}$ & $m_{8 i}$ & $m_{9 i}$ & $m_{10 i}$ & $m_{11 i}$ & $m_{12 i}$ & $m_{13 i}$ & $m_{14 i}$ & $m_{15 i}$ & $m_{16 i}$ \\
\hline 1 & 1 & 1 & 0 & $\frac{29}{4}$ & 1 & 1 & 0 & $\frac{29}{4}$ & 0 & 1 & 0 & 1 & 0 & $\frac{29}{4}$ & 0 & $\frac{29}{4}$ \\
2 & 1 & 0 & 1 & $\frac{29}{4}$ & 1 & -1 & 0 & 0 & $\frac{29}{4}$ & 0 & 1 & 0 & -1 & $\frac{29}{4}$ & 0 & $-\frac{29}{4}$ \\
3 & 1 & -1 & 0 & $\frac{29}{4}$ & 1 & 1 & 0 & $-\frac{29}{4}$ & 0 & -1 & 0 & -1 & 0 & $\frac{29}{4}$ & 0 & $\frac{29}{4}$ \\
4 & 1 & 0 & -1 & $\frac{29}{4}$ & 1 & -1 & 0 & 0 & $-\frac{29}{4}$ & 0 & -1 & 1 & 1 & $\frac{29}{4}$ & 0 & $-\frac{29}{4}$ \\
5 & 1 & 6 & 0 & 36 & 36 & 36 & 0 & 216 & 0 & 216 & 0 & 216 & 0 & 1296 & 0 & 1296 \\
6 & 1 & 0 & 6 & 36 & 36 & -36 & 0 & 0 & 216 & 0 & 216 & 0 & -216 & 1296 & 0 & -1296 \\
7 & 1 & -6 & 0 & 36 & 36 & 36 & 0 & -216 & 0 & -216 & 0 & 216 & 0 & 1296 & 0 & 1296 \\
8 & 1 & 0 & -6 & 36 & 36 & -36 & 0 & 0 & -216 & 0 & -216 & 0 & 216 & 1296 & 0 & -1296 \\
9 & 1 & $\sqrt{2}$ & $\sqrt{2}$ & 4 & 4 & 0 & 2 & $4 \sqrt{2}$ & $4 \sqrt{2}$ & $4 \sqrt{2}$ & $4 \sqrt{2}$ & 0 & 0 & 16 & 8 & 0 \\
10 & 1 & $-\sqrt{2}$ & $\sqrt{2}$ & 4 & 4 & 0 & -2 & $-4 \sqrt{2}$ & $4 \sqrt{2}$ & $-4 \sqrt{2}$ & $4 \sqrt{2}$ & 0 & 0 & 16 & -8 & 0 \\
11 & 1 & $-\sqrt{2}$ & $-\sqrt{2}$ & 4 & 4 & 0 & 2 & $-4 \sqrt{2}$ & $-4 \sqrt{2}$ & $-4 \sqrt{2}$ & $-4 \sqrt{2}$ & 0 & 0 & 16 & 8 & 0 \\
12 & 1 & $\sqrt{2}$ & $-\sqrt{2}$ & 4 & 4 & 0 & -2 & $4 \sqrt{2}$ & $-4 \sqrt{2}$ & $4 \sqrt{2}$ & $-4 \sqrt{2}$ & 0 & 0 & 16 & -8 & 0 \\
13 & 1 & $\frac{3}{\sqrt{2}}$ & $\frac{3}{\sqrt{2}}$ & 9 & 9 & 0 & $\frac{9}{2}$ & $\frac{27}{\sqrt{2}}$ & $\frac{27}{\sqrt{2}}$ & $\frac{27}{\sqrt{2}}$ & $\frac{27}{\sqrt{2}}$ & 0 & 0 & 81 & $\frac{81}{2}$ & 0 \\
14 & 1 & $-\frac{3}{\sqrt{2}}$ & $\frac{3}{\sqrt{2}}$ & 9 & 9 & 0 & $-\frac{9}{2}$ & $-\frac{27}{\sqrt{2}}$ & $\frac{27}{\sqrt{2}}$ & $-\frac{27}{\sqrt{2}}$ & $\frac{27}{\sqrt{2}}$ & 0 & 0 & 81 & $-\frac{81}{2}$ & 0 \\
15 & 1 & $-\frac{3}{\sqrt{2}}$ & $-\frac{3}{\sqrt{2}}$ & 9 & 9 & 0 & $\frac{9}{2}$ & $-\frac{27}{\sqrt{2}}$ & $-\frac{27}{\sqrt{2}}$ & $-\frac{27}{\sqrt{2}}$ & $-\frac{27}{\sqrt{2}}$ & 0 & 0 & 81 & $\frac{81}{2}$ & 0 \\
16 & 1 & $\frac{3}{\sqrt{2}}$ & $-\frac{3}{\sqrt{2}}$ & 9 & 9 & 0 & $-\frac{9}{2}$ & $\frac{27}{\sqrt{2}}$ & $-\frac{27}{\sqrt{2}}$ & $\frac{27}{\sqrt{2}}$ & $-\frac{27}{\sqrt{2}}$ & 0 & 0 & 81 & $-\frac{81}{2}$ & 0 \\
\hline \hline
\end{tabular}

$\hat{\mathbf{f}}^{e q}=\left(\rho, j_{x}, j_{y}, e^{\prime}, \hat{f}_{5}^{e q}, \hat{f}_{6}^{e q}, \cdots, \hat{f}_{16}^{e q}\right)^{T}$, where $\hat{f}_{5}^{e q}=2 P+\left(j_{x}^{2}+j_{y}^{2}\right) / \rho, \hat{f}_{6}^{e q}=\left(j_{x}^{2}-j_{y}^{2}\right) / \rho$, $\hat{f}_{7}^{e q}=j_{x} j_{y} / \rho, \hat{f}_{8}^{e q}=\left(e^{\prime}+2 P\right) j_{x} / \rho, \hat{f}_{9}^{e q}=\left(e^{\prime}+2 P\right) j_{y} / \rho, \hat{f}_{10}^{e q}=\left(4 P+j_{x}^{2} / \rho+j_{y}^{2} / \rho\right) j_{x} / \rho$, $\hat{f}_{11}^{e q}=\left(4 P+j_{x}^{2} / \rho+j_{y}^{2} / \rho\right) j_{y} / \rho, \hat{f}_{12}^{e q}=\left(2 P+j_{x}^{2} / \rho-j_{y}^{2} / \rho\right) j_{x} / \rho, \hat{f}_{13}^{e q}=\left(-2 P+j_{x}^{2} / \rho-j_{y}^{2} / \rho\right) j_{y} / \rho$, $\hat{f}_{14}^{e q}=2(b+2) \rho R^{2} T^{2}+(6+b) R T\left(j_{x}^{2}+j_{y}^{2}\right) / \rho+\left(j_{x}^{2}+j_{y}^{2}\right)^{2} / \rho^{3}, \hat{f}_{15}^{e q}=\left[(b+4) P+\left(j_{x}^{2}+j_{y}^{2}\right) / \rho\right] j_{x} j_{y} / \rho^{2}$, $\hat{f}_{16}^{e q}=\left[(b+4) P+\left(j_{x}^{2}+j_{y}^{2}\right) / \rho\right]\left(j_{x}^{2}-j_{y}^{2}\right) / \rho^{2}$, where $P=\rho R T$, and $e^{\prime}=b \rho R T+j_{\alpha}^{2} / \rho$ is the twice of total energy e. The recovered Navier-Stokes equations are as follows:

$$
\frac{\partial \rho}{\partial t}+\frac{\partial j_{x}}{\partial x}+\frac{\partial j_{y}}{\partial y}=0
$$




$$
\begin{aligned}
& \frac{\partial j_{x}}{\partial t}+\frac{\partial}{\partial x}\left(\frac{j_{x}^{2}}{\rho}\right)+\frac{\partial}{\partial y}\left(\frac{j_{x} j_{y}}{\rho}\right) \\
& =-\frac{\partial P}{\partial x}+\frac{\partial}{\partial y}\left[\frac{\rho R T}{s_{7}}\left(\frac{\partial u_{y}}{\partial x}+\frac{\partial u_{x}}{\partial y}\right)\right] \\
& +\frac{\partial}{\partial x}\left[\frac{\rho R T}{s_{5}}\left(1-\frac{2}{b}\right)\left(\frac{\partial u_{x}}{\partial x}+\frac{\partial u_{y}}{\partial y}\right)+\frac{\rho R T}{s_{6}}\left(\frac{\partial u_{x}}{\partial x}-\frac{\partial u_{y}}{\partial y}\right)\right] \text {, } \\
& \frac{\partial j_{y}}{\partial t}+\frac{\partial}{\partial x}\left(\frac{j_{x} j_{y}}{\rho}\right)+\frac{\partial}{\partial y}\left(\frac{j_{y}^{2}}{\rho}\right) \\
& =-\frac{\partial P}{\partial y}+\frac{\partial}{\partial x}\left[\frac{\rho R T}{s_{7}}\left(\frac{\partial u_{y}}{\partial x}+\frac{\partial u_{x}}{\partial y}\right)\right] \\
& +\frac{\partial}{\partial y}\left[\frac{\rho R T}{s_{5}}\left(1-\frac{2}{b}\right)\left(\frac{\partial u_{x}}{\partial x}+\frac{\partial u_{y}}{\partial y}\right)-\frac{\rho R T}{s_{6}}\left(\frac{\partial u_{x}}{\partial x}-\frac{\partial u_{y}}{\partial y}\right)\right], \\
& \frac{\partial e}{\partial t}+\frac{\partial}{\partial x}\left[(e+P) j_{x} / \rho\right]+\frac{\partial}{\partial y}\left[(e+P) j_{y} / \rho\right] \\
& =\frac{\partial}{\partial x}\left\{\frac{\rho R T}{s_{8}}\left[\left(\frac{b}{2}+1\right) R \frac{\partial T}{\partial x}+\left(2 \frac{\partial u_{x}}{\partial x}-\frac{2}{b} \frac{\partial u_{x}}{\partial x}-\frac{2}{b} \frac{\partial u_{y}}{\partial y}\right) u_{x}+\left(\frac{\partial u_{y}}{\partial x}+\frac{\partial u_{x}}{\partial y}\right) u_{y}\right]\right\} \\
& +\frac{\partial}{\partial y}\left\{\frac{\rho R T}{s_{9}}\left[\left(\frac{b}{2}+1\right) R \frac{\partial T}{\partial y}+\left(2 \frac{\partial u_{y}}{\partial y}-\frac{2}{b} \frac{\partial u_{x}}{\partial x}-\frac{2}{b} \frac{\partial u_{y}}{\partial y}\right) u_{y}+\left(\frac{\partial u_{y}}{\partial x}+\frac{\partial u_{x}}{\partial y}\right) u_{x}\right]\right\} .
\end{aligned}
$$

When $s_{5}=s_{6}=s_{7}=s_{8}=s_{9}$, the above Navier-Stokes equations reduce to

$$
\begin{gathered}
\frac{\partial \rho}{\partial t}+\frac{\partial j_{\alpha}}{\partial x_{\alpha}}=0, \\
\frac{\partial j_{\alpha}}{\partial t}+\frac{\partial\left(j_{\alpha} j_{\beta} / \rho\right)}{\partial x_{\beta}}=-\frac{\partial P}{\partial x_{\alpha}}-\frac{\partial}{\partial x_{\beta}} P_{\alpha \beta}^{\prime}, \\
\frac{\partial e}{\partial t}+\frac{\partial}{\partial x_{\alpha}}\left[(e+P) u_{\alpha}\right]=\frac{\partial}{\partial x_{\beta}}\left(\left(\frac{b}{2}+1\right) \mu R \frac{\partial T}{\partial x_{\beta}}-P_{\alpha \beta}^{\prime} u_{\alpha}\right),
\end{gathered}
$$

where

$$
\begin{gathered}
\mu=\frac{\rho R T}{s}, \mu_{B}=(2 / 3-2 / b) \frac{\rho R T}{s} \\
P_{\alpha \beta}^{\prime}=-\mu\left(\frac{\partial u_{\alpha}}{\partial x_{\beta}}+\frac{\partial u_{\beta}}{\partial x_{\alpha}}-\frac{2}{3} \frac{\partial u_{\chi}}{\partial x_{\chi}} \delta_{\alpha \beta}\right)-\mu_{B} \frac{\partial u_{\chi}}{\partial x_{\chi}} \delta_{\alpha \beta},(\alpha, \beta, \gamma=x, y) .
\end{gathered}
$$

Similar to the case of the first MRT model, the second one works also well for shocked compressible fluids. For more general cases, similar modifications to the collision operators of the moments related to the energy flux should be made [45]. 


\section{SIMULATIONS ON HYDRODYNAMIC INSTABILITIES}

Hydrodynamic instabilities are ubiquitous in natural and industrial processes. The Rayleigh-Taylor(RT) instability, Richtmyer-Meshkov(RM) instability and Kelvin-Helmholtz (KH) instability are highly concerned in weapon physics and inertial confinement fusion. For example, during the spherical implosion procedure, the high pressure applied at the outside of the shell drives a very strong shock wave towards the centre of the device. This shock wave first accelerates the interface to a high velocity. Towards the end of the implosion the interface is decelerated by a combination of shock waves reflected from the center of the device and continuous deceleration due to build up of high pressure in the thermonuclear material. Such a very complicated acceleration/deceleration behavior results in two processes, RT instability and RM instability. Since the implosion is generally not perfectly symmetrical, the shear at the interface induces the third process, KH instability. Hydrodynamic instabilities in such procedure influence significantly the implosion physics and weapon performance. In this section, we summarize our recent attempts on LB simulations on KH [51] and RM

instabilities [42, 43]. When studying the RM instability, the system must be compressible. In the case of KH instability, the system can be compressible or nearly incompressible. As a first step, we attempted the case with nearly incompressible fluids.

\section{A. Richtmyer-Meshkov instability}

The RM instability arises when a shock wave interacts with an interface separating two different fluids. It combines various compressible phenomena, such as shock interaction and refraction, with hydrodynamic instability, including nonlinear growth and subsequent transition to turbulence, across a wide range of Mach numbers. In inertial confinement fusion, the RM instability causes mixing between the capsule material and the fuel within, limiting final compression and thus the ability to achieve energy break-even or production. The RM instability problems in the plane occur when a shock wave travels from a light medium to a heavy one or when the shock wave travels from a heavy medium to a light one. 


\section{Shock wave from light to heavy media}

A practical example for this case is that the shock wave travels from air to $S F_{6}$. For such a case, in our LB simulations we set the following initial physical field,

$$
\left\{\begin{array}{c}
\left(\rho, u_{x}, u_{y}, p\right)_{l}=(1.34161,0.361538,0,1.51332) \\
\left(\rho, u_{x}, u_{y}, p\right)_{m}=(1,0,0,1) \\
\left(\rho, u_{x}, u_{y}, p\right)_{r}=(5.04,0,0,1)
\end{array}\right.
$$

where the subscripts $l, m, r$ indicate the left, middle, right regions of the whole domain. Such an initial configuration can be explained as below: the interface of the middle and right regions separates the light and heavy media; the interface of the left and middle regions is the shock front; the shocked light medium is in the left and the pre-shocked is in the middle regions. Initially, the two media have the same pressure and different densities and temperatures. The corresponding Mach number of the shock wave traveling from left is 1.2. The shock wave will hit the interface with an initial sinusoidal perturbation. The initial sinusoidal perturbation at the interface reads $x=0.25 \times N_{x} \times d x+0.008 \times \cos (20 \pi y)$, where the cycle in $y$-direction of initial perturbation is 0.1 , the amplitude is $0.008, N_{x}$ is grid number, and $d x$ is grid size. The following boundary conditions are imposed: (i) inflow at the left side; (ii) outflow at the right side, and (3) periodic in the $y$-directions. $\gamma=1.4$ in the whole domain.

Since the Mach number is 1.2, the compressibility effects in this case is not negligible. Figure 7 shows the density and pressure contours at four different times, $t=0,0.06,0.3$ and 1.15. When the shock wave passes the interface from the left, a reflected shock wave to the left and a transmission wave to the right are generated (clearly seen in pressure field at $t=0.06$ ). The transmission wave has a certain curvature at this time. Due to the compression, the interface produces a small deformation, and the perturbation amplitude reduces slightly. At $t=0.3$, the reflected shock wave has been out of the computational domain, and the transmission wave becomes flat, which is consistent with the theoretical analysis of [52]. The perturbation amplitude begins to increase under the pressure gradient, producing the bubble and spike structures. The misalignment of pressure and density gradients causes a deposition of vorticity at the top of spike structure, and a mushroom shape is formed eventually. Fig.8 shows the changes of perturbation amplitude and growth rate with time. The amplitude is defined as half of the maximum distance between the crest and trough. 


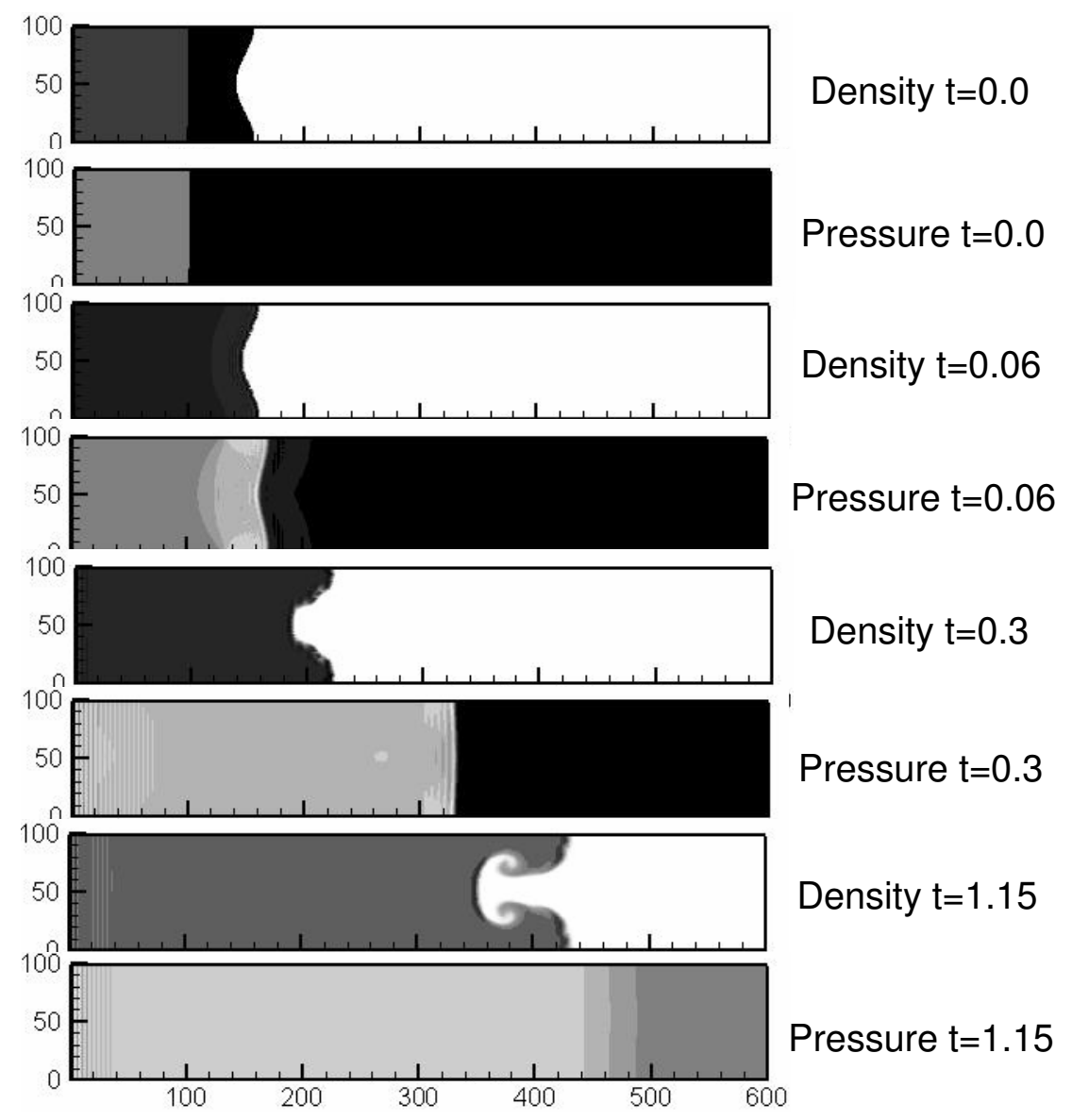

FIG. 7: Snapshots of RM instability (from light to heavy medium): density and pressure contours at $t=0, t=0.06, t=0.3, t=1.15$, respectively. From deep to light color, the level corresponds to the increase of values.

From Fig.8 one can clearly find the initial decrease of perturbation amplitude. During this initial period, the growth rate is negative.

Now we go to some theories to explain and validate the simulation results. Richtmyer [53] proposed an impulsive model in the case of a reflected shock wave via modifying the linear theory of Taylor for Rayleigh-Taylor instability. According to the impulse model, the growth rate reads,

$$
\frac{d a}{d t}=k \Delta u A_{1} a_{1}, \quad a_{1}=a_{0}\left(1-\frac{\Delta u}{D}\right)
$$

where $k=2 \pi / \lambda$ is the wave number, $\Delta u$ is the velocity change across the interface, $A_{1}$ is the post-shock Atwood number, $a_{1}$ represents the post-shock amplitude, $a_{0}$ is the initial amplitude, $D$ denotes the incident shock speed. $C m p r=1-\Delta u / D$ is compression ratio. According to the initial conditions, the solution is $C m p r=0.84, d a / d t=0.063$. In the 

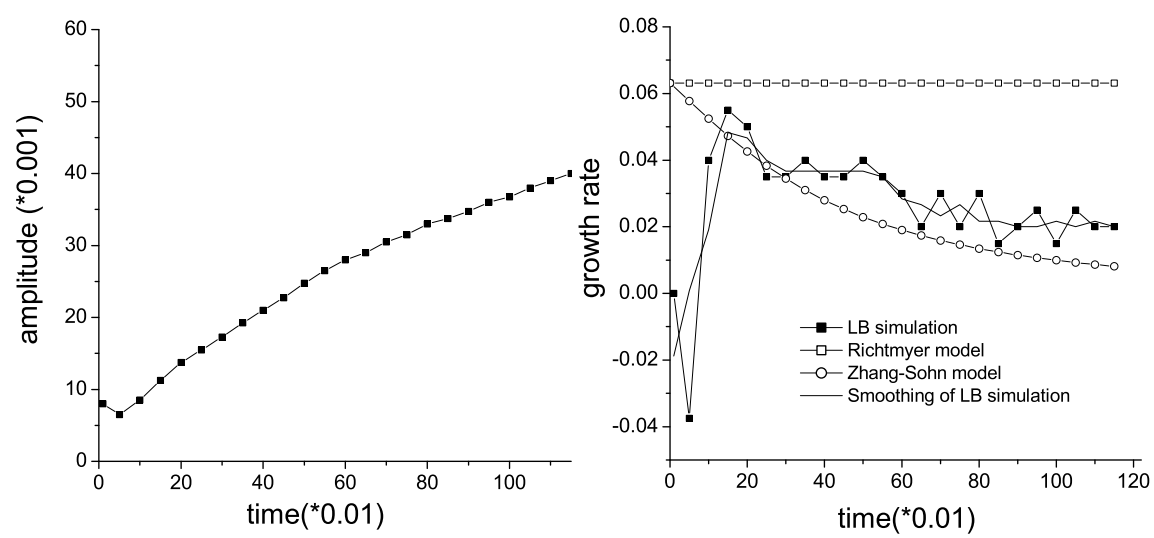

FIG. 8: Amplitude and growth rate changes with time (from light to heavy medium).

experiments of Meshkov [54] and Benjamin [55], the measured growth rates are only about one half of that predicted by the impulsive model. Zhang and Sohn [56] developed a model for the growth of RM unstable interface from early to late times in the case of light-heavy transition. The amplitude growth reads

$$
\frac{d a}{d t}=\frac{v_{0}}{1+k^{2} v_{0} a_{1} t+\max \left[0,\left(k a_{1}\right)^{2}-\left(A_{1}\right)^{2}+0.5\right]\left(k v_{0} t\right)^{2}}
$$

where $v_{0}=k \Delta u A_{1} a_{1}$. As shown in Fig.8, the LB result for growth rate qualitatively agrees well with that of Zhang-Sohn model. The amplitude reaches the minimum value 0.0065 at time $t=0.05$, so the compression ratio obtained in simulation is $C m p r=0.0065 / 0.008=$ 0.81. By the least squares fitting, the growth rate of amplitude 0.03 is obtained, which is about one half of the growth rate predicted by the impulsive model and consequently is in good agreement with the experimental result. In the nonlinear stage, the simulation results agree qualitatively well with the perturbation model proposed by Zhang and Sohn.

\section{Shock wave from heavy to light media}

A practical example is that the shock wave travels from air to $H e$. To better understand such a case, in our LB simulation, we set a planar shock wave with the Mach number 2.5 impinging on a sinusoidal perturbation $x=0.1+0.008 \times \cos (20 \pi y)$, where the cycle and amplitude of initial perturbation are the same with the case where shock wave travels from 
light to heavy media. The initial physical field is as below:

$$
\left\{\begin{array}{c}
\left(\rho, u_{x}, u_{y}, p\right)_{l}=(3.33333,2.07063,0,7.125) \\
\left(\rho, u_{x}, u_{y}, p\right)_{m}=(1,0,0,1) \\
\left(\rho, u_{x}, u_{y}, p\right)_{r}=(0.138,0,0,1)
\end{array}\right.
$$

The boundary conditions in the $y$-direction and at the left side are the same as the case where shock wave travels from light to heavy media. Two different boundaries are applied at the right side: outflow condition (case I) and reflecting boundary (case II). The computational domain is a rectangle $0.6 \times 0.1$ for case $\mathrm{I}$ and $0.3 \times 0.1$ for case II, respectively.

Figure 9 shows the simulation results for density field. Figure (a) corresponds to the outflow boundary and figure (b) corresponds to the reflecting boundary. Here $\gamma=1.4$. The collision parameters in case I are $s_{5}=10^{4}, 10^{5}$ for the others, and in case II are $s_{5}=10^{3}, 10^{5}$ for the others. Simulation results show the following physical procedure: When the shock wave passes the interface, a reflected rarefaction wave to the left and a transmission wave to the right are generated. The pressure of heavy fluid near the crest is greater than the light fluid pressure. Driven by the pressure gradient, the perturbation amplitude decreases with the interface motion to the right. Then, the peak and valley of initial interface invert, the heavy and light fluids gradually penetrate into each other as time goes on, the light fluid "rises" to form a bubble and the heavy fluid "falls" to generate a spike. In case I, the transmission wave continues to move to the right, and no longer interacts with the interface. The disturbance of the interface continues to grow, eventually forming a mushroom shape. In case II, the transmission wave reaches the solid wall on the right and reflects to the left, encounters the interface again. This is known as the "reshocking" process. Following reshocking, the interface is compressed, as seen from the kink in the bubble. Furthermore, the amplitude grows more rapidly than prior to reshocking, the increased growth is due to the additional vorticity deposited on the evolving interface during reshocking. The pressure contours and velocity vectors near the interface at time $t=0.08$ are shown in Fig.10. Figure 11 shows the change of disturbance amplitudes with time, corresponding to case I and case II, respectively. Because of the reshocking effect, a significant difference between Fig.11(a) and Fig.11(b) can be observed.

The interface reversal phenomenon is observed in the second case. With the interaction between shock wave and interface, disturbance will grow continuously. In the early stage, 

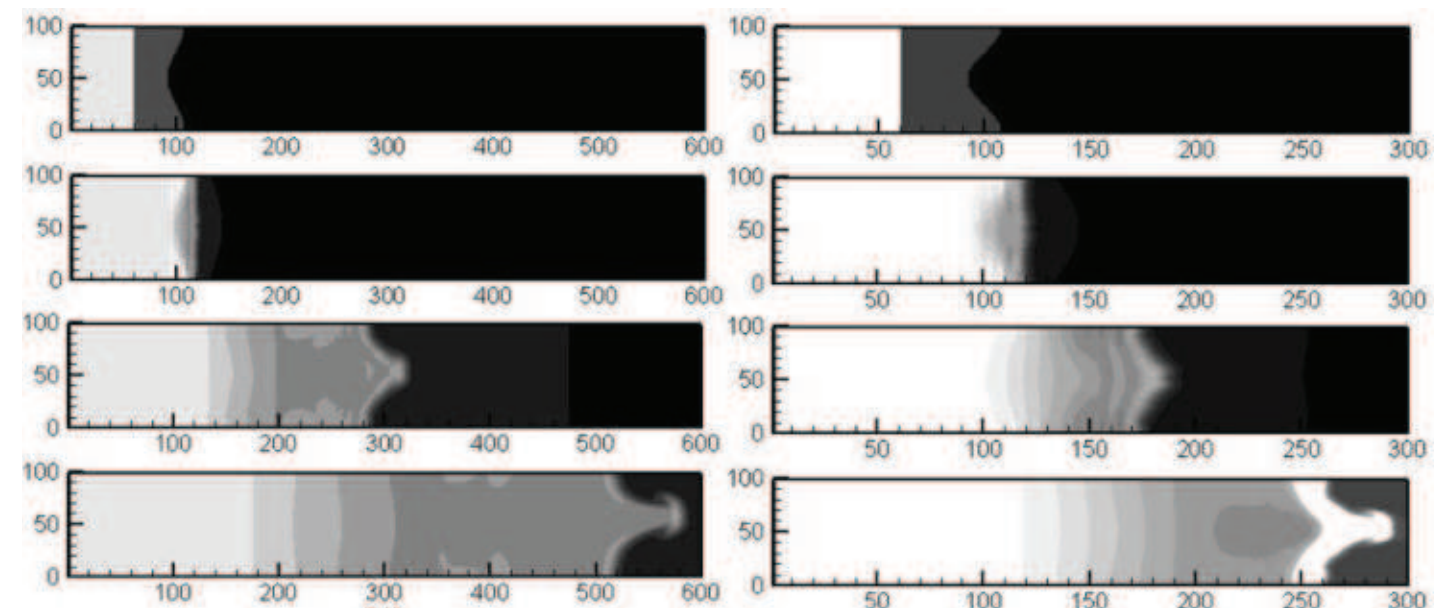

(a)

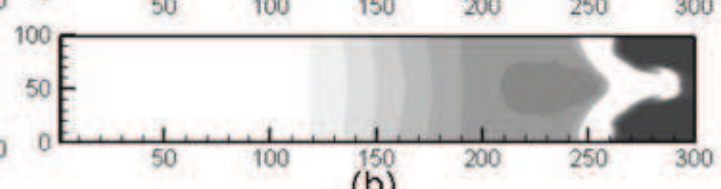

(b)

FIG. 9: Snapshots of RM instability (from heavy to light medium). (a) Outflow boundary. From top to bottom, $t=0,0.02,0.08,0.16$, respectively. (b) Reflecting boundary. From top to bottom, $t=0,0.02,0.04,0.08$, respectively. From deep to light color, the level corresponds to the increase of density.
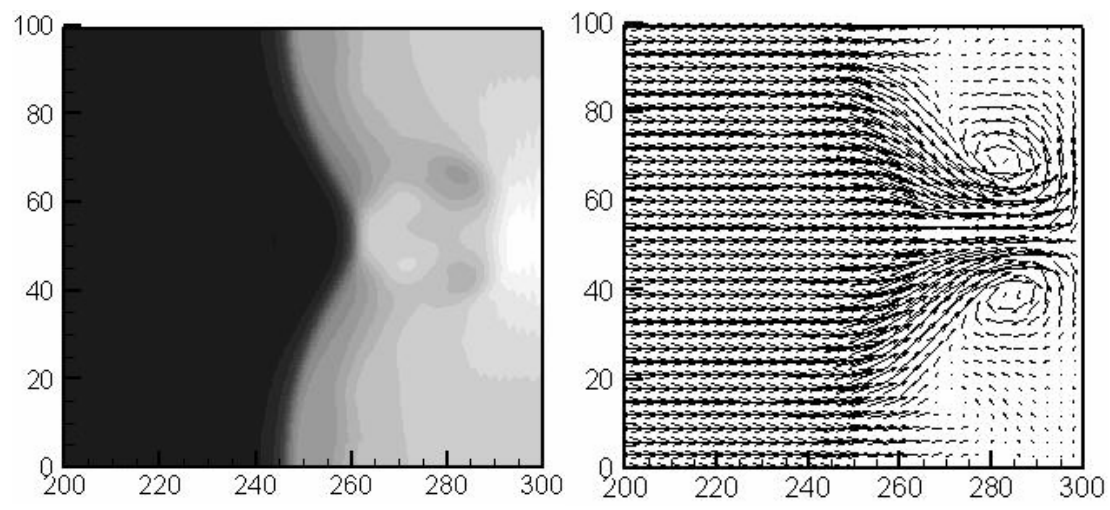

FIG. 10: Pressure contours and velocity vectors at time $t=0.08$ (from heavy to light medium, reflecting boundary). From deep to light color, the level corresponds to the increase of pressure.

logarithm of growth rate is nearly linear with time, while changes into the non-linear in the late stage, spikes and bubbles occur.

\section{B. Kelvin-Helmholtz instability}

During the later stage KH instability strengthens the nonlinear developing of RT and RM instabilities, enhances the small scale mixing. In some cases, it may break the spkies. But in 

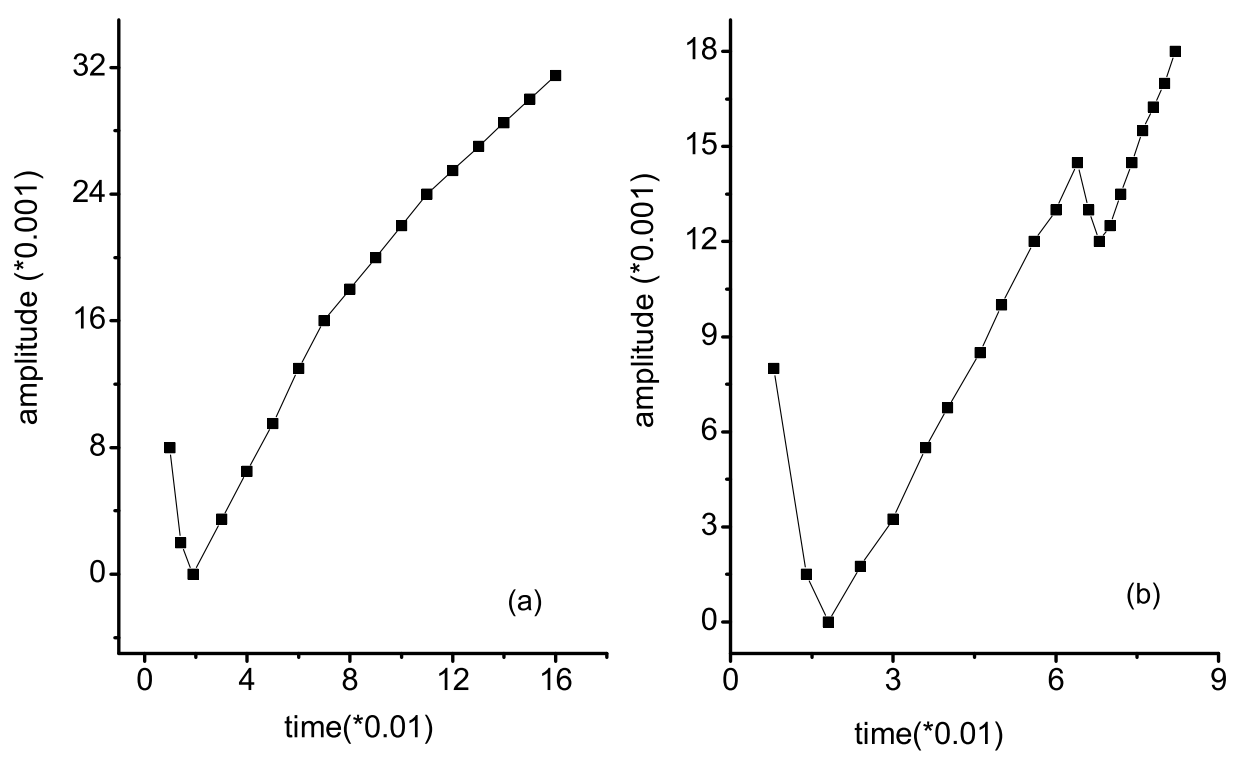

FIG. 11: Amplitude change with time (from heavy to light medium). (a) Outflow boundary, (b) Reflecting boundary.

some cases, we failed to observe the full effects of KH instability. For example, in the Eagle Nebula, why has the famous "Pillars of Creation" so large scale structures, instead of being broken by many small scale vortices? There must be some mechanisms to restrain the $\mathrm{KH}$ instability. Therefore, people study the KH instability from two sides. How does the KH instability evolve? How to enhance or restrain the KH instability? The strong nonlinearity and multiscale interactions make difficult theoretical study. The very complex $3 \mathrm{D}$ behavior challenge experimental diagnosis. Our LB modeling and simulation aim to help understand better the KH instability from both the two sides.

For investigating the Kelvin-Helmholtz instability, we set the following initial physical field,

$$
\begin{gathered}
\rho(x)=\frac{\rho_{L}+\rho_{R}}{2}-\frac{\rho_{L}-\rho_{R}}{2} \tanh \left(\frac{x}{D_{\rho}}\right), \\
v(x)=\frac{v_{L}+v_{R}}{2}-\frac{v_{L}-v_{R}}{2} \tanh \left(\frac{x}{D_{v}}\right), \\
P_{L}=P_{R}=P,
\end{gathered}
$$

where we have two characteristic length scales, $D_{\rho}$ and $D_{v}$, which are the widths of density and velocity transition layers, respectively. $\rho_{L}=5.0\left(\rho_{R}=2.0\right)$ is the density away from the interface of the left (right) fluid. $v_{L}=0.5\left(v_{R}=-0.5\right)$ is the velocity away from the 
interface in $y$-direction of the left (right) fluid, and $P_{L}\left(P_{R}\right)=2.5$ is the pressure in the left (right) side. The system can be approximately thought of as "incompressible". The whole calculation domain is a rectangle with length 0.6 and height 0.2 , which is divided into $600 \times 200$ uniform meshes. A simple velocity perturbation in the $x$-direction is introduced to trigger the $\mathrm{KH}$ rollup and it is in the following form

$$
u=u_{0} \sin (k y) \exp (-k x),
$$

where $u_{0}=0.02$ is the amplitude of the perturbation. Here, $k$ is the wave number of the initial perturbation, and is set to be $10 \pi$. The time step is $\Delta t=10^{-5}$.

At the initial linear increasing stage of KH INSTABILITY, the amplitude $\eta$ of perturbation evolves according to the following relation, $\eta=\eta_{0} e^{\gamma t}$, where $\gamma$ is the growth coefficient and is dependent on the gradient of tangential velocity and gradient of density around the interface. In other words, $\gamma$ is dependent on the width of velocity transition layer $D_{v}$ and width of density transition layer $D_{\rho}$. We discuss separately the KHI in three cases, (i) $D_{v}$ is variable and $D_{\rho}$ is fixed, (ii) $D_{\rho}$ is variable and $D_{v}$ is fixed, (iii) both $D_{\rho}$ and $D_{v}$ are variable. The increasing rate $\gamma$ for cases (i), (ii) and (iii) are referred to as $\gamma_{v}, \gamma_{\rho}$ and $\gamma_{R}$, respectively. We numerically obtain $\gamma_{v}, \gamma_{\rho}$ and $\gamma_{R}$ via fitting the curves of $\left.\ln E_{x}\right|_{\max }(t)$ versus the time $t$, where $\left.E_{x}\right|_{\max }(t)$ is the maximum of $E_{x}(x, y, t)$ in the whole computational domain, $E_{x}(x, y, t)=\rho(x, y, t) u^{2}(x, y, t) / 2$ is the perturbed kinetic energy at the position $(x$, $y)$ at each time step $t$.

Although viscosity damps the evolution of the KH INSTABILITY, here we focus on cases such as in inertial confined fusion where effects of the viscosity are generally negligible. Therefore, throughout the simulations, $\tau$ is set to be $10^{-5}$ to reduce the physical viscosity. Boundary conditions are as below. Periodic in the $y$-direction and outflow (zero gradient) in the $x$-direction.

\section{Velocity gradient effect}

Figure 12 shows the evolution of the density field for the case with $D_{v}=4$ and $D_{\rho}=8$ at four different times. At $t=0.3$ the interface has been wiggling under the initial perturbation

and velocity shear. A nicely rolled vortex occurs and develops around the initial interface after the initial linear growth stage. The vortex becomes larger with time and a mixing layer 


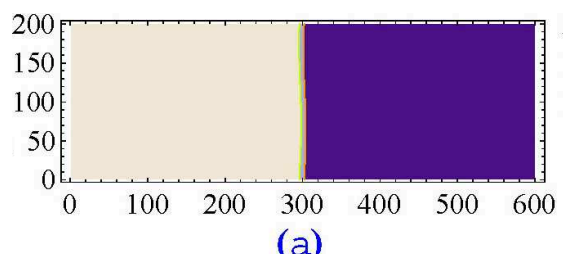

(a)

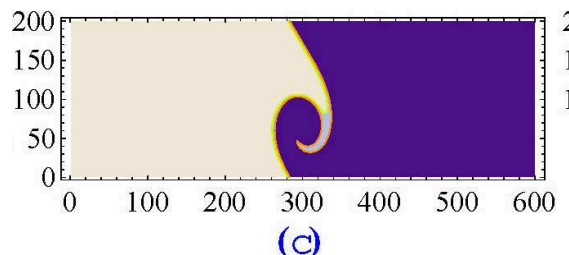

(c)

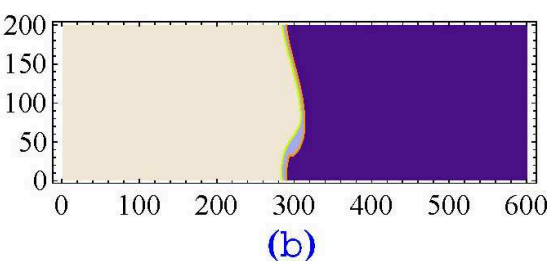

(b)

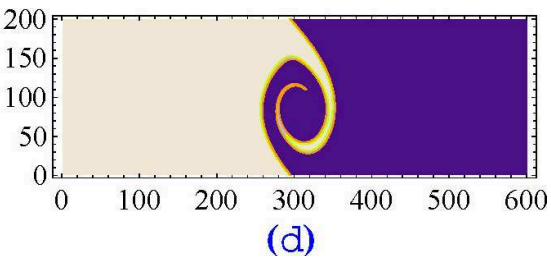

FIG. 12: (Color online) Density evolutions of KH INSTABILITY simulated using the LB model, where $D_{v}=4$ and $D_{\rho}=8, t=0.1$ in (a), $t=0.3$ in (b), $t=0.5$ in (c), and $t=0.7$ in (d).

forms around the initial interface.

To investigate the velocity gradient effect, we fix the width of the density transition layer. Figure 13 shows the density field for various $D_{v}$ at the same time, where $D_{\rho}=8, t=0.6$ and $D_{v}=4,8,12,16$ in (a)-(d), respectively. Five contour lines are plotted in each plot. It is clear that the width of the velocity transition layer significantly affects the evolution of $\mathrm{KH}$ instability. The larger the value of $D_{v}$, the weaker the $\mathrm{KH}$ instability, and the later the vortex appears. In Figs.(a) and (b), large vortices have been formed demonstrating that the evolution is embarking on the nonlinear stage. While in Figs. (c) and (d), the evolution is in the weakly nonlinear stage. Figures (a)-(d) show that a wider velocity transition zone is helpful for stabilizing the $\mathrm{KH}$ instability.

The peak kinetic energy $\left.E_{x}\right|_{\max }$ partly indicates the interacting strength of two different fluids. Figure 14(a) shows that logarithm of $\left.E_{x}\right|_{\max }$ versus time. The initial state shows a linear behavior. The slope $k$ increases with decreasing the width $D_{v}$. After the initial stage, $\ln \left(\left.E_{x}\right|_{\max }\right)$ approaches a saturation value via a nonlinear growth stage. During the initial linear stage, we have $E_{x} \propto u^{2} \propto\left(e^{\gamma t}\right)^{2}$. So, the slope $k$ here can be used to calculate the growth coefficient $\gamma$ in the linear growth stage, $k=2 \gamma$. The logarithm of $\gamma$ decreases linearly with $D_{v}$ [see Fig.14(b)]. Our LB results confirm the theoretical analysis of Wang, et al. [57]. In the classical case, the linear growth rate is $\gamma_{c}=k \sqrt{\rho_{1} \rho_{2}}\left(v_{1}-v_{2}\right) /\left(\rho_{1}+\rho_{2}\right) \propto \Delta v$, where $\Delta v$ is the shear velocity difference. A wider transition layer decreases the local or the effective shear velocity difference $\Delta v$, which results in a smaller linear growth rate and a longer linear growth time. 

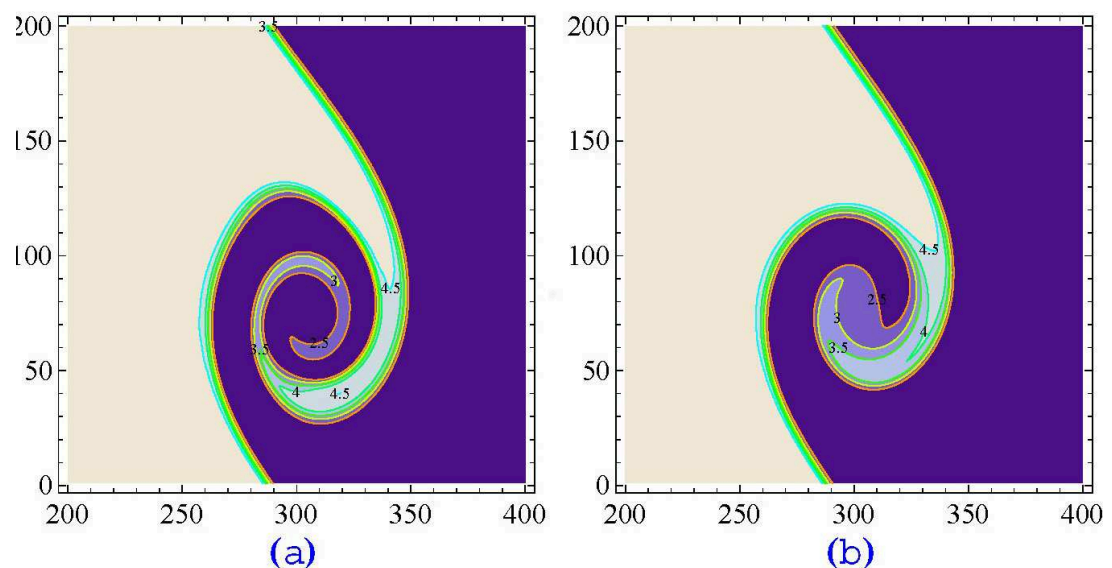

(b)
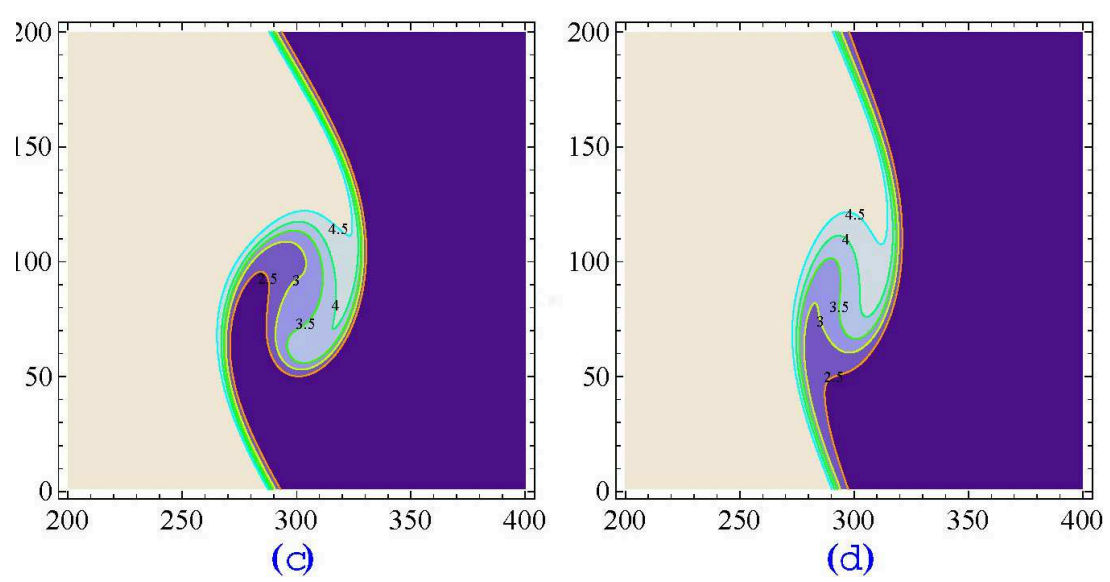

FIG. 13: (Color online) Vortices in the mixing layer as a function of $D_{v}$ at $t=0.6$, where $D_{v}=4$ in (a), $D_{v}=8$ in (b), $D_{v}=12$ in (c), and $D_{v}=16$ in (d). The density transition layer $D_{\rho}$ is fixed to be 8 .
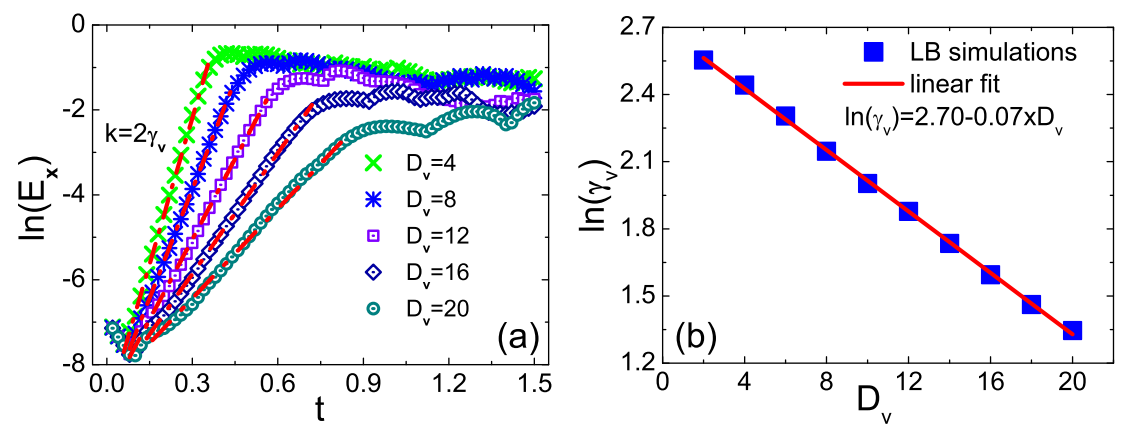

FIG. 14: (Color online) (a) Time evolution of the perturbed peak kinetic energy $\left.E_{x}\right|_{\max }$ along the $x$-axis in ln-linear scale for various widths of velocity transition layer. The dash-dotted lines represent the linear fits to the initial linear growth regimes. (b) Linear growth rate as a function of the width $D_{v}$ of velocity transition layer. 

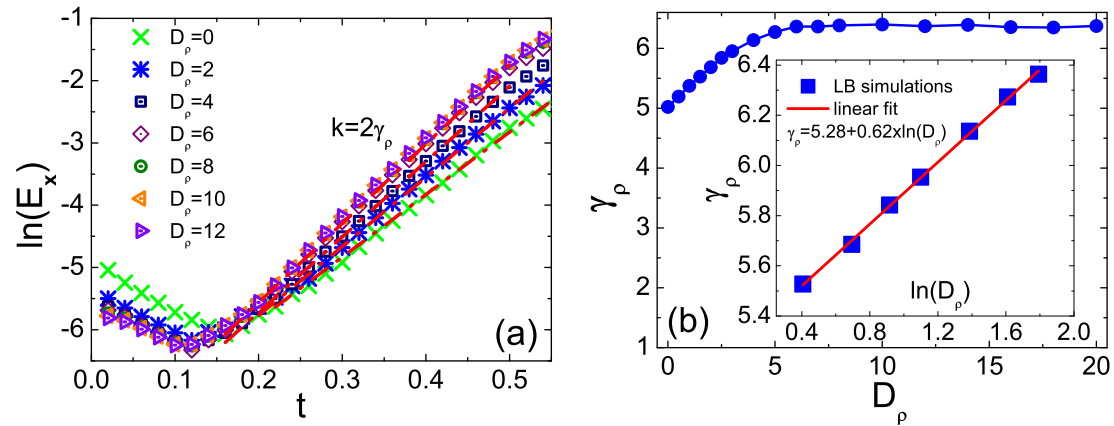

FIG. 15: (Color online) (a) Time evolution of the logarithm of the peak kinetic energy $\left.E_{x}\right|_{\max }$ along the $x$-axis for various widths of density transition layers. (b) Linear growth rate as a function of the width $D_{\rho}$ of density transition layer.

\section{Density gradient effect}

The density gradient effect is investigated in a similar way. Here $D_{v}$ is fixed. The initial conditions are described as, $\left(\rho_{L}, v_{L}, P_{L}\right)=(5.0,0.5,1.5)$ and $\left(\rho_{R}, v_{R}, P_{R}\right)=(1.25$, $-0.5,1.5)$. Figure $15(\mathrm{a})$ shows evolution of the logarithm of peak kinetic energy $\left.E_{x}\right|_{\max }$ along the $x$-axis versus time $t$ for various widths of density transition layers. Here $D_{v}=2$, $\Delta x=\Delta y=0.002, \Delta t=10^{-5}$. Results for $D_{\rho}=0,2,4,6,8,10$, and 12 are shown. For fixed width of velocity transition layer and density difference, the linear growth rate first increases with the width $D_{\rho}$. But when $D_{\rho}$ is large than a critical value which is about 6, it does not vary significantly any more [see Fig.15(b) ]. During the linear growth stage, $\gamma_{\rho}$ increases linearly with the logarithm of $D_{\rho}$. Figures 14 and 15 indicate the effective interaction width of $D_{\rho}$ is less than that of $D_{v}$. The LB results here confirm also the theoretical analysis of Wang, et al. [57]. In the classical case, the square of the linear growth rate is $\gamma_{c}^{2}=k^{2} \rho_{1} \rho_{2}\left(v_{1}-v_{2}\right)^{2} /\left(\rho_{1}+\rho_{2}\right)^{2} \propto\left(1-A^{2}\right) \Delta v^{2}$, where $A=\left(\rho_{1}-\rho_{2}\right) /\left(\rho_{1}+\rho_{2}\right)$ is the Atwood number. A wider density transition zone reduces the Atwood number around the interface. Then in the process of exchanging momentum in the direction normal to the interface, the perturbation can obtain more energy from the shear kinetic energy than in cases with sharper interfaces. Therefore, a thinner density transition layer is helpful to restrain the $\mathrm{KH}$ instability. 


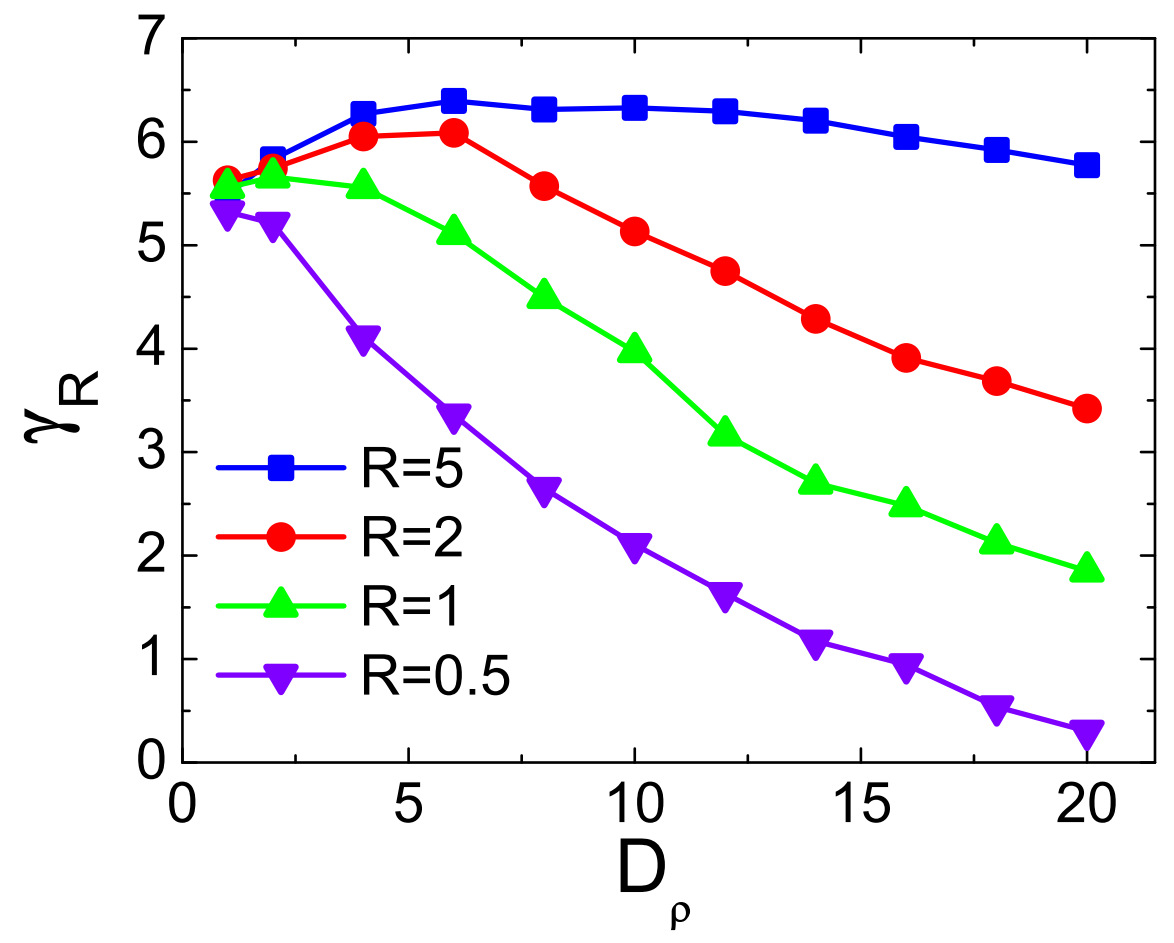

FIG. 16: (Color online) The linear growth rate versus the width of density transition layer for $R=0.5,1,2$, and 5 . The initial density, shear velocity and pressure of the two fluids are $\left(\rho_{L}, v_{L}\right.$, $\left.P_{L}\right)=(5.0,0.5,1.5)$ and $\left(\rho_{R}, v_{R}, P_{R}\right)=(1.25,-0.5,1.5)$.

\section{Hybrid effects of velocity and density gradients}

In practical systems, at the interface of two fluids with a tangential velocity difference, both the velocity and the density gradients exist. There is a competition between effects of the two kinds of gradients. We introduce a coefficient $R=D_{\rho} / D_{v}$ through which we analyze the combined effects. The linear growth rate versus $D_{\rho}$ under various values of $R$ is shown in Fig.16. Here $R=0.5,1,2$, and 5 , as shown in the legend. On the whole, the hybrid effect of the two kinds of gradients is to reduce the linear growth rate $\gamma_{R}$. Only at small $D_{\rho}$ and when $R>1$, the hybrid effect makes larger the linear growth rate. This indicates again that the effective interaction width of the velocity transition layer $D_{v}^{E}$ is wider than that of density transition layer $D_{\rho}^{E}$. 


\section{CONCLUSIONS}

Both the LB and the hydrodynamic equations are simplified dynamic models of practical systems. Compared with the latter, the former puts the physical modeling on a more fundamental level. When numerically study a physical procedure, the working dynamic model is not the one evolving continuously in space and time but the one discretized in the code. Improving the discrete template and reasonably adding viscosity term are in fact some remedies to the working dynamic model. Compared with the LB based on BGK approximation, the MRT-LB introduces a new framework where various physical modes can be considered separately. The developed SRT-LB and MRT-LB are complementary from the sides of convenience and applicability. Compared with the hydrodynamic descriptions, both the SRT-LB and MRT-LB present new measurements for the deviations of systems from their thermodynamic equilibria. The LB model is being extended to study the compressibility effects, effects of shocking and detonation, thermal effects on the hydrodynamic instabilities [51] and multiphase flows [58 60], etc., which are all-important issues in science and engineering.

\section{Acknowledgements}

The authors thank Prof. Guoxi Ni for many helpful discussions. AX and GZ acknowledge support of the Science Foundations of CAEP [under Grant Nos. 2012B0101014 and 2011A0201002]. AX, GZ, YG and XY acknowledge support of National Natural Science Foundation of China [under Grant Nos. 11075021, 11171038, 11202003 and 91130020]. YG acknowledges support of Technology Support Program of LangFang [under Grant Nos. 2010011030 and $201101118 / 21 / 23 / 24]$.

[1] S. Succi, The Lattice Boltzmann Equation for Fluid Dynamics and Beyond, Oxford University Press, New York, (2001).

[2] S. Chen, H. Chen, D. Martnez, and W. Matthaeus, Phys. Rev. Lett. 67 (1991) 3776.

[3] G. Vahala, B. Keating, M. Soe, J. Yepezand, and L. Vahala, Comm. Comp. Phys. 4 (2008) 624 . 
[4] A. Ladd, J. Fluid Mech. 271 (1994) 311.

[5] A. Gunstensen, D. Rothman, S. Zaleski, and G. Zanetti, Phys. Rev. A 43 (1991) 4320.

[6] X. Shan and H. Chen, Phys. Rev. E 47 (1993) 1815.

[7] M. Swift, W. Osborn, and J. Yeomans, Phys. Rev. Lett. 75 (1995) 830.

[8] X. He, S. Chen, and R. Zhang, J. Comput. Phys. 152 (1999) 642.

[9] A. Xu, G. Gonnella, and A. Lamura, Phys. Rev. E 67 (2003) 056105.

[10] A. Xu, G. Gonnella, and A. Lamura, Phys. Rev. E 74 (2006) 011505.

[11] A. Xu, G. Gonnella, A. Lamura, G. Amati, and F. Massaioli, Europhys. Lett. 71 (2005) 651.

[12] M. Sbragaglia, R. Benzi, L. Biferale, S. Succi, K. Sugiyama, and F. Toschi, Phys. Rev. E 75 (2007) 026702.

[13] V. Sofonea, A. Lamura, G. Gonnella, and A. Cristea, Phys. Rev. E 70 (2004) 046702.

[14] A. Cristea, G. Gonnella, A. Lamura, and V. Sofonea, Commun. Comput. Phys. 7 (2010) 350.

[15] S. Succi, E. Foti, and F. Higuera, Europhys. Lett. 10 (1989) 433.

[16] Y. Xu, Y. Liu, X. Yang, and F. Wu, Commun. Theor. Phys. 49 (2008) 1319.

[17] W. Fickett and W. C. Davis, Detonation theory and experiment, Dover publications, INC., New York, (1979).

[18] C. Wang, X. Zhang, C. W. Shu, and J. Ning, J. Comput. Phys. 231, 653 (2012).

[19] S. Tan, C. Wang, C. W. Shu, and J. Ning, J. Comput. Phys. 231, 2510 (2012).

[20] F. J. Alexander, H. Chen, S. Chen and G. D. Doolen, Phys. Rev. A 46, 1967 (1992).

[21] G. W. Yan, Y. S. Chen, S. X. Hu, Phys. Rev. E 59, 454 (1999).

[22] C. H. Sun, Phys. Rev. E 58, 7283 (1998).

[23] C. Sun and A. T. Hsu, Phys. Rev. E 68, 016303 (2003).

[24] T. Kataoka and M. Tsutahara, Phys. Rev. E 69, 056702 (2004).

[25] T. Kataoka and M. Tsutahara, Phys. Rev. E 69, 035701(R)(2004).

[26] M. Watari and M. Tsutahara, Phys. Rev. E 67 (2003) 036306.

[27] M. Watari and M. Tsutahara, Phys. Rev. E 70, 016703 (2004).

[28] M. Watari, Physica A 382, 502 (2007).

[29] A. Xu, Europhys. Lett. 69, 214 (2005).

[30] A. Xu, Phys. Rev. E 71, 066706 (2005).

[31] S. Ansumali, I.V. Karlin, and J. Stat. Phys. 107, 291 (2002).

[32] S. Ansumali, I.V. Karlin, and H.C. Ottinger, Europhys. Lett. 63, 798 (2003). 
[33] Y. Li, R. Shock, R. Zhang, and H. Chen, J. Fluid Mech. 519, 273 (2004).

[34] V. Sofonea, A. Lamura, G. Gonnella, A. Cristea, Phys. Rev. E 70, 046702 (2004).

[35] X.F. Pan, A.G. Xu, G.C. Zhang, and S. Jiang, Int. J. Mod. Phys. C 18, 1747 (2007).

[36] Y. Gan, A. Xu, G. Zhang, X. Yu, and Y. Li, Physica A 387, 1721 (2008).

[37] Y. Gan, A. Xu, G. Zhang, and Y. Li, Commun. Theor. Phys. 50, 201 (2008).

[38] F. Chen, A. Xu, G. Zhang, Y. Gan, C. Tao, and Y. Li, Commun. Theor. Phys. 52, 681 (2009).

[39] F. Chen, A. Xu, G. Zhang, Y. Li, Commun. Theor. Phys. 54, 1121, (2010).

[40] Y. Gan, A. Xu, G. Zhang, and Y. Li, Commun. Theor. Phys. 56, 490 (2011).

[41] F. Chen, A. Xu, G.Zhang, Y. Li, Phys. Lett. A 375, 2129 (2011).

[42] F. Chen, A. Xu, G.Zhang, Y. Li, S. Succi, EuroPhys. Lett. 90, 54003 (2010).

[43] F. Chen, A. Xu, G.Zhang, Y. Li, Commun. Theor. Phys. 55, 325 (2011).

[44] F. Chen, A. Xu, G. Zhang, Y. Li, Commun. Theor. Phys. 56, 333, (2011).

[45] F. Chen, A. Xu, G.Zhang, Y. Li, Theroe. \& Appl. Mech. Lett. 1, 052004 (2011).

[46] F. J. Higuera, S. Succi and R. Benzi, Europhys. Lett. 9, 345 (1989);

[47] F. J. Higuera, and J. Jimenez, Europhys. Lett. 9, 662 (1989).

[48] P. Lallemand and L. S. Luo, Phys. Rev. E 61, 6546 (2000).

[49] P. Lallemand and L. S. Luo, Phys. Rev. E 68, 036706 (2003).

[50] P. Lallemand, D. d'Humières, L.S. Luo, and R.Rubinstein, Phys. Rev. E 467, 021203 (2003).

[51] Y. Gan, A. Xu, G. Zhang, and Y. Li, Phys. Rev. E 83, 056704(2011).

[52] A. L. Velikovich, J. P. Dahlburg, Schmitt, Phys. Plasmas 7, 1662 (2000).

[53] R. D. Richtmyer, Comm. Pure Appl. Math. 8 (1960) 297.

[54] E. E. Meshkov, Sov. Fluid Dyn. 4, 101 (1969).

[55] R. F. Benjamin, Advances in Compressible Turbulent Mixing, edited by W. P. Dannevik, A. C. Buckingham, and C. E. Leith (1992).

[56] Q. Zhang and S. Sohn, Phys. Fluids 9, 1106 (1997).

[57] L. F. Wang, W. H. Ye, and Y. J. Li, Phys. Plasma 17, 042103 (2010).

[58] Y. Gan, A. Xu, G. Zhang, Y. Li and H. Li, Phys. Rev. E 84, 046715 (2011).

[59] Y. Gan, A. Xu, G. Zhang, and Y. Li, Europhys. Lett. 97, 44002 (2012).

[60] Y. Gan, A. Xu, G. Zhang, and Y. Li, Front. Phys. DOI.10.1007/s11467-012-0245-0 (2012). 